Indian J. Pure Appl. Math., 51(2): 631-657, June 2020

(c) Indian National Science Academy

DOI: $10.1007 / \mathrm{s} 13226-020-0421-9$

\title{
ESTIMATION OF EXPONENTIATED NADARAJAH-HAGHIGHI DISTRIBUTION UNDER PROGRESSIVELY TYPE-II CENSORED SAMPLE WITH APPLICATION TO BLADDER CANCER DATA
}

\author{
Ziyad A. Alhussain* and Essam A. Ahmed** \\ * Department of Mathematics, College of Science in Al-Zulfi, \\ Majmaah University, Saudi Arabia \\ ** Department of Administrative and Financial Sciences, Taibah University, \\ Community College of Khyber 41941, Saudi Arabia \\ and \\ Mathematics Department, Sohag University, Sohag 82524, Egypt \\ e-mails: Z.alhussain@mu.edu.sa,emohammed@taibahu.edu.sa, \\ esam_alsyd@yahoo.ccom \\ (Received 2 March 2017; after final revision 16 October 2017; \\ accepted 24 March 2019)
}

In this article we consider statistical inferences about the unknown parameters of the exponentiated Nadarajah-Haghighi (ENH) distribution based on progressively type-II censoring using classical and Bayesian procedures. For classical procedures, maximum likelihood (ML) and least square estimators of the unknown parameters are derived. The Bayes estimators are obtained based on both the symmetric (squared error) and asymmetric (LINEX, general entropy) loss functions. Furthermore, Markov chain Monte Carlo (MCMC) technique is used to compute the Bayes estimators and the associated credible intervals. Moreover, asymptotic confidence intervals are constructed using the normality property of the ML estimates. After that, the asymptotic confidence intervals using ML estimates and two parametric bootstrap confidence intervals are provided to compare with Bayes credible intervals. Finally, simulation study and a bladder cancer application are presented to illustrate the proposed methods of estimation.

Key words : Exponentiated Nadarajah-Haghighi distribution; maximum likelihood estimators; least squares estimators; Bayes estimators; bootstrap; Markov chain Monte Carlo

2010 Mathematics Subject Classification : 62F05, 62F10, 62F15, 62F40, 65C40. 


\section{INTRODUCTION}

Many of the scientific researches and books have talked about the exponential distribution and its applications. It is the most widely used lifetime model in reliability theory, because of its simplicity and mathematical feasibility. Recently some generalizations of the exponential distribution are proposed with the hope that they attract wider applications in many fields like engineering, economics, physics, biological sciences, among others. These generalizations models can be defined by adding parameters to an existing distribution, see for example generalized exponential distribution proposed by Gupta and Kundu (1999) and exponentiated exponential distribution considered by Gupta and Kundu (2001).

By adding a parameter to exponential model, an extension of the exponential distribution, named as exponential extension model has been proposed by Nadarajah and Haghighi (2011). The two parameter exponential extension model represent the shape and scale parameter. It is observed that this family always has a decreasing probability function like an exponential model but it allows for increasing, decreasing and constant hazard rates like a Weibull model or an exponentiated exponential model. It can be used as an alternative to the gamma, Weibull and exponentiated exponential distributions. However, it does not provide a reasonable parametric fit for modelling data with bathtub-shaped and unimodal failure rate function. These cases are quite common in reliability and biological studies.

The lifetime distributions with bathtub shaped hazard rate functions have attracted the attention of many researchers as the lifetimes of various industrial items including electrical and mechanical products, as well as survival times of various biological entities exhibit such characteristics (see for instance, Rajarshi and Rajarshi, 1988). After, Hjorth (1980) proposed a three-parameter distribution with increasing, decreasing, constant or bathtub-shaped failure rate function, more of authors introduced new distributions for modelling bathtub-shaped. For example, a three-parameter model, named exponentiated Weibull distribution, was introduced by Mudholkar and Srivastave (1993). Another three-parameter model was proposed by Marshall and Olkin (1997) and called extended Weibull distribution. Xie et al. (2002) proposed a three-parameter modified Weibull extension with a bathtub shaped hazard function. Another generalization of the Weibull distribution is the modified Weibull distribution discussed in Lai et al. (2003). Zhang and Xie (2007) addressed the extended Weibull distribution in Marshall and Olkin (1997) by introducing an additional parameter to an existing model and also investigated some properties of this distribution and studied the versatility of the model in modelling failure data. Some models for lifetime data have been proposed very recently such as in Silva et al. (2010), Liu and Wang (2013) and El-Gohary et al. (2013). 
Lemonte (2013) proposed a new generalization of the exponential extension distribution the called as the exponentiated Nadarajah-Haghighi distribution by adding an extra parameter form in Nadarajah-Haghighi distribution. He has established several interesting properties of this distribution and also observed that the hazard function of it can be constant, increasing, decreasing, upside down bathtub or bathtub-shaped depending on the shape parameters. To define ENH distribution formally; if $X \sim \operatorname{ENH}(\alpha, \lambda, \beta)$, then the probability density function (PDF) of $X$ is

$$
f_{X}(x)=\alpha \beta \lambda(1+\lambda x)^{\alpha-1} e^{1-(1+\lambda x)^{\alpha}}\left(1-e^{1-(1+\lambda x)^{\alpha}}\right)^{\beta-1}, x>0, \alpha>0, \lambda>0, \beta>0
$$

where the parameters $\alpha>0$ and $\beta>0$ control the shapes of the distribution, and the parameter $\lambda>0$ is the scale parameter. The corresponding cumulative distribution function (CDF) is

$$
F_{X}(x)=\left(1-e^{1-(1+\lambda x)^{\alpha}}\right)^{\beta}, x>0, \alpha>0, \lambda>0, \beta>0 .
$$

The reliability and hazard rate functions are, respectively,

$$
S(t)=1-\left(1-e^{1-(1+\lambda t)^{\alpha}}\right)^{\beta}, t>0,
$$

and

$$
h(t)=\frac{\alpha \beta \lambda(1+\lambda x)^{\alpha-1} e^{1-(1+\lambda x)^{\alpha}}\left(1-e^{1-(1+\lambda t)^{\alpha}}\right)^{\beta-1}}{1-\left(1-e^{1-(1+\lambda x)^{\alpha}}\right)^{\beta}}, t>0 .
$$

The ENH distribution is motivated by its usefulness in exploring tail properties and also for improving the goodness-of-fit. Depending on the parameters $\alpha$ and $\beta$ the hazard rate function $h(t)$ can be constant (when $\alpha=\beta=1$ ), monotonically decreasing (when $\alpha<1$ and $\beta<1$ ), monotonically increasing (when $\alpha>1$ and $\beta>1$ ), upside down bathtub (unimodal) (when, $\alpha<1$ and $\beta>1$ ) or bathtub-shaped (when $\alpha>1 \beta<1$ ). The parameter $\lambda$ does not change the shape of the failure rate function since it is a scale parameter.Another current reason for using ENH distribution is its applications in lifetime data analysis. Also, the ENH distribution includes the exponential distribution, the generalized exponential distribution (Gupta and Kundu, 1999) and the extended exponential distribution (Nadarajah and Haghighi, 2011) as special sub-models.

To obtain knowledge about product lifetime distribution, life-testing experiments are run at different product development and testing stages before the product can be put on the market. When the product are extremely reliable or the cost of a failing a product is high, a censoring scheme is always imposed in the life-testing experiment in order to save time and cost of the experiment. One of the commonly used censoring schemes is the type-II right censoring scheme whereas the lifetesting experiment is terminated as soon as the $m$ th (where $m$ is pre-fixed) failure is observed. Then, 
only the first $m$ failures out of $n$ units under test will be observed. The data obtained from such a restrained life-test will be referred to as a type-II censored sample. A generalization of the type-II censoring scheme, called progressive type-II censoring scheme, has been proposed in the literature. It allows one to remove live units from the test at various stages during the experiment that can be used in other tests, saving the experimenter cost and time consumption while still allowing for observing extreme data. Under this scheme of censoring, from a total of $n$ units placed on a life-test, only $m$ failures $(m<n)$ are completely observed. At the time $x_{1: m: n}$ of first failure, a random sample of size $R_{1}$ is immediately drawn and removed from the $(n-1)$ survivals units, hence leaving $n-1-R_{1}$ survival items. Similarly, at the time $x_{2: m: n}$ of the second failure, $R_{2}$ of $n-2-R_{1}$ surviving units are removed from the experiment. Finally, at the time $x_{m: m: n}$ of the $m$ th failure, all the remaining $R_{m}=n-m-R_{1}-\ldots-R_{m-1}$ surviving units are removed from the experiment. The ordered failure times $X_{1: m: n} \leq X_{2: m: n} \leq \ldots \leq X_{m: m: n}$ arising from such a progressively type-II right censored sample are called progressively type-II censored order statistics. Notice that if $R_{1}=R_{2}=\ldots=R_{m-1}=0$, then $R_{m}=n-m$, consequently this scheme reduces to the conventional type-II right censoring scheme. Also, this scheme includes as special cases the complete sample situation (when $R_{1}=R_{2}=\ldots=R_{m}=0$ and $n=m$ ). For details on progressive Type-II censoring, we refer to Balakrishnan and Aggarwala (2000), Balakrishnan (2007) or in the excellent book by Balakrishnan and Cramer (2014).

To illustrate this model by a practical example (see Singh et al. (2014)), consider that a doctor performs an experiment with $n$ bladder cancer patients with remission times (in months). Because of expense of reduction times (in months) the experiment is terminated after a prefixed number of bladder cancer patients and we get type-II censored data. After type-II censoring another situation of bladder cancer patients with remission times (in months) may arise, the first bladder cancer patient has died due to some other unanticipated circumstances such as heart assault, accident, damage of lever, depletion of funds, etc.; some patients leave the experiment and go for treatment to other doctor/hospital. Similarly, after the second death a few more leave and so on. Finally, the doctor stops taking observation as soon as the predetermined number of deaths (say $m$ ) is recorded. Which has arise a scenario of progressive type-II censoring. Recently, several articles have been published on estimating the parameters for different distribution functions, see, for example, Soliman et al. (2012), Ahmed $(2014,2015)$ and the references cite therein.

To the best of our knowledge, the estimation problem, where the parameters $\alpha, \lambda$ and $\beta$ of ENH distribution are unknown, has not been studied in the literature under progressively censored samples. In this article, we study this problem from both classical and Bayesian point of view. It is not possible 
to compute the exact distributions of the ML estimates based on progressively type-II right censored . We have used the asymptotic distribution of the ML estimates to construct approximate confidence intervals of the unknown parameters. While the method of ML estimation is the most popular in terms of theoretical prospective, the LS estimation method is computational easier to deal with the estimation parameters. Further; we construct confidence intervals of the unknown parameters, based on the bootstrapping method. Bayesian estimates of unknown quantities are derived under different symmetric and asymmetric loss functions such as squared error, LINEX and general entropy. It is observed that the Bayes estimators cannot be obtained in closed forms. We have used the Markov chain Monte Carlo (MCMC) technique to compute the Bayes estimates and also to construct the credible intervals.

The rest of the paper is presented as follows: The ML and LS estimates of the unknown parameters are considered in Section 2. Also; in this section asymptotic confidence intervals are constructed for unknown parameters using the normality property of corresponding ML estimates. Bootstrap confidence intervals are discussed in Section 3. In Section 4, Bayes estimates are obtained with respect to squared error, LINEX and general entropy loss functions. Markov Chain Monte Carlo (MCMC) samples are further utilized to construct credible intervals for unknown parameters. In the fifth section, an application of the ENH distribution to remission times of bladder cancer patients is given. Section 6 is described numerical experiments via simulation study. Finally, concluding remarks are given in Section 7.

\section{Classical Estimation Procedures}

\subsection{ML estimates}

The likelihood function based on progressively type-II censored data $x=\left(x_{1}, x_{2}, \ldots, x_{m}\right)$ from $\operatorname{ENH}(\alpha, \lambda, \beta)$ distribution, with censoring scheme $R=\left(R_{1}, R_{2}, \ldots, R_{m}\right)$, is specified as the following form

$L(\underline{x} ; \alpha, \lambda, \beta)=C(\alpha \beta \lambda)^{m} e^{-\sum_{i=1}^{m}\left(1+\lambda x_{i}\right)^{\alpha}} \prod_{i=1}^{m} \frac{\left(1+\lambda x_{i}\right)^{\alpha-1}\left[1-\left(1-e^{1-\left(1+\lambda x_{i}\right)^{\alpha}}\right)^{\beta}\right]^{R_{i}}}{\left(1-e^{1-\left(1+\lambda x_{i}\right)^{\alpha}}\right)^{1-\beta}}$

For $a>0$ a real non-integer, we use the series representation

$$
(1-z)^{a}=\sum_{k=0}^{\infty}\left(\begin{array}{l}
a \\
k
\end{array}\right)(-1)^{k} z^{k},|z|<1,
$$


We thus have:

$$
\begin{aligned}
L(\underline{x} ; \alpha, \lambda, \beta)= & C(\alpha \beta \lambda)^{m} e^{-\sum_{i=1}^{m}\left(1+\lambda x_{i}\right)^{\alpha}} \prod_{i=1}^{m}\left(1+\lambda x_{i}\right)^{\alpha-1} \sum_{k=0}^{\infty}\left(\begin{array}{c}
R_{i} \\
k
\end{array}\right)(-1)^{k} \\
& \left(1-e^{1-\left(1+\lambda x_{i}\right)^{\alpha}}\right)^{\beta(k+1)-1},
\end{aligned}
$$

where $C=n\left(n-R_{1}-1\right)\left(n-R_{1}-R_{2}-2\right) \ldots\left(n-\sum_{i=1}^{m-1}\left(R_{i}+1\right)\right)$.

The corresponding log-likelihood function is

$$
\begin{aligned}
\ell= & \log (C)+m \log (\alpha)+m \log (\lambda)+m \log (\beta)+(\alpha-1) \sum_{i=1}^{m} \log \left(1+\lambda x_{i}\right)-\sum_{i=1}^{m}\left(1+\lambda x_{i}\right)^{\alpha} \\
& +\sum_{i=1}^{m} \log \left[\sum_{k=0}^{\infty}\left(\begin{array}{c}
R_{i} \\
k
\end{array}\right)(-1)^{k}\left(1-e^{1-\left(1+\lambda x_{i}\right)^{\alpha}}\right)^{\beta(k+1)-1}\right] .
\end{aligned}
$$

The derivatives of (6) with respect to $\alpha, \lambda$ and $\beta$ are given by

$$
\begin{aligned}
\frac{\partial \ell}{\partial \alpha}= & \frac{m}{\alpha}-\sum_{i=1}^{m}\left(1+\lambda x_{i}\right) \log \left(1+\lambda x_{i}\right)+\sum_{i=1}^{m} e^{1-\left(1+\lambda x_{i}\right)^{\alpha}}\left(1+\lambda x_{i}\right)^{\alpha} \log \left(1+\lambda x_{i}\right) \\
& \times\left[\frac{\sum_{k=0}^{\infty}\left(\begin{array}{c}
R_{i} \\
k
\end{array}\right)(-1)^{k}[\beta(k+1)-1]\left(1-e^{1-\left(1+\lambda x_{i}\right)^{\alpha}}\right)^{\beta(k+1)-2}}{\sum_{k=0}^{\infty}\left(\begin{array}{c}
R_{i} \\
k
\end{array}\right)(-1)^{k}\left(1-e^{1-\left(1+\lambda x_{i}\right)^{\alpha}}\right)^{\beta(k+1)-1}}\right]+\sum_{i=1}^{m} \log \left(1+\lambda x_{i}\right),
\end{aligned}
$$

and

$$
\begin{aligned}
& \frac{\partial \ell}{\partial \lambda}= \frac{m}{\lambda}+(\alpha-1) \sum_{i=1}^{m} \frac{x_{i}}{\left(1+\lambda x_{i}\right)}-\alpha \sum_{i=1}^{m} x_{i}\left(1+\lambda x_{i}\right)^{\alpha-1}+\sum_{i=1}^{m} e^{1-\left(1+\lambda x_{i}\right)^{\alpha}}\left(1+\lambda x_{i}\right)^{\alpha-1} \\
& \times\left[\frac{\sum_{k=0}^{\infty}\left(\begin{array}{c}
R_{i} \\
k
\end{array}\right)(-1)^{k} \alpha x_{i}[\beta(k+1)-1]\left(1-e^{1-\left(1+\lambda x_{i}\right)^{\alpha}}\right)^{\beta(k+1)-2}}{\sum_{k=0}^{\infty}\left(\begin{array}{c}
R_{i} \\
k
\end{array}\right)(-1)^{k}\left(1-e^{\left.1-\left(1+\lambda x_{i}\right)^{\alpha}\right)^{\beta(k+1)-1}}\right]}\right] \\
& \frac{\partial \ell}{\partial \beta}=\frac{m}{\beta}+\sum_{i=1}^{m}\left[\frac{\sum_{k=0}^{\infty}\left(\begin{array}{c}
R_{i} \\
k
\end{array}\right)(-1)^{k}\left(1-e^{1-\left(1+\lambda x_{i}\right)^{\alpha}}\right)^{\beta(k+1)-1} \log \left(1-e^{1-\left(1+\lambda x_{i}\right)^{\alpha}}\right)^{(k+1)}}{\sum_{k=0}^{\infty}\left(\begin{array}{c}
R_{i} \\
k
\end{array}\right)(-1)^{k}\left(1-e^{1-\left(1+\lambda x_{i}\right)^{\alpha}}\right)^{\beta(k+1)-1}}\right]
\end{aligned}
$$

The ML estimates $\hat{\alpha}_{M}, \hat{\lambda}_{M}$ and $\hat{\beta}_{M}$ can be obtained by setting Equations (7)-(9) to zero and solving them numerically. Here, the Newton-Raphson iteration method is used to obtain the parameter estimates. The algorithm is comprised of the following steps: 
Step 1 : Use the method of moments or some other proper estimates of the parameters as starting point of iteration, denote the estimates as $\left(\alpha_{0}, \lambda_{0}, \beta_{0}\right)$ and set $k=0$. where

Step 2: Calculate $\left(\frac{\partial \ell}{\partial \alpha}, \frac{\partial \ell}{\partial \lambda}, \frac{\partial \ell}{\partial \beta}\right)_{\alpha_{k}, \lambda_{k}, \beta_{k}}$ and the observed Fisher information matrix $I\left(\alpha_{0}, \lambda_{0}, \beta_{0}\right)$,

$$
I(\alpha, \lambda, \beta)=\left(\begin{array}{ccc}
-\frac{\partial^{2} \ell}{\partial \alpha^{2}} & -\frac{\partial^{2} \ell}{\partial \alpha \partial \lambda} & -\frac{\partial^{2} \ell}{\partial \alpha \partial \beta} \\
-\frac{\partial^{2} \ell}{\partial \lambda \partial \alpha} & -\frac{\partial^{2} \ell}{\partial \lambda^{2}} & -\frac{\partial^{2} \ell}{\partial \lambda \partial \beta} \\
-\frac{\partial^{2} \ell}{\partial \beta \partial \alpha} & -\frac{\partial^{2} \ell}{\partial \beta \partial \lambda} & -\frac{\partial^{2} \ell}{\partial \beta^{2}}
\end{array}\right)
$$

The expressions for the elements of matrix (10) are given in Appendix.

Step 3 : Update $(\alpha, \lambda, \beta)$ as

$$
\left(\alpha_{k+1}, \lambda_{k+1}, \beta_{k+1}\right)=\left(\alpha_{k}, \lambda_{k}, \beta_{k}\right)+\left(\frac{\partial \ell}{\partial \alpha}, \frac{\partial \ell}{\partial \lambda}, \frac{\partial \ell}{\partial \beta}\right)_{\alpha_{k}, \lambda_{k}, \beta_{k}} \times I^{-1}\left(\alpha_{k}, \lambda_{k}, \beta_{k}\right)
$$

Step $4:$ Set $k=k+1$, and then go back to step 1 .

Step 5 : Continue the iterative steps until $\left|\left(\alpha_{k+1}, \lambda_{k+1}, \beta_{k+1}\right)-\left(\alpha_{k}, \lambda_{k}, \beta_{k}\right)\right|$ is smaller than a threshold value $\epsilon$. The final estimates of $(\alpha, \lambda, \beta)$ are the MLE of the parameters, denoted as $\left(\hat{\alpha}_{M}, \hat{\lambda}_{M}, \hat{\beta}_{M}\right)$.

Since, the exact distribution of ML estimates are not easy to obtain, one can use the concept of large sample theory to obtain the confidence intervals (CIs). The approximate variances and the CIs of the parameters are obtained by inverting th observed Fisher matrix $I(\alpha, \lambda, \beta)$. That is

$$
I^{-1}\left(\hat{\alpha}_{M}, \hat{\lambda}_{M}, \hat{\beta}_{M}\right)=\left(\begin{array}{ccc}
\operatorname{var}\left(\hat{\alpha}_{M}\right) & \operatorname{cov}\left(\hat{\alpha}_{M}, \hat{\lambda}_{M}\right) & \operatorname{cov}\left(\hat{\alpha}_{M}, \hat{\beta}_{M}\right) \\
& \operatorname{var}\left(\hat{\lambda}_{M}\right) & \operatorname{cov}\left(\hat{\lambda}_{M}, \hat{\beta}_{M}\right) \\
& & \operatorname{var}\left(\hat{\beta}_{M}\right)
\end{array}\right)
$$

The diagonal elements of $I^{-1}\left(\hat{\alpha}_{M}, \hat{\lambda}_{M}, \hat{\beta}_{M}\right)$ provides the asymptotic variances for the parameters $\alpha, \lambda$ and $\beta$ respectively.

By using asymptotic normal (AN) distribution of MLEs $\hat{\alpha}_{M}, \hat{\lambda}_{M}$ and $\hat{\beta}_{M}$, the $100(1-\gamma) \%$ AN CIs for $\alpha, \lambda$ and $\beta$ are constructed by $\left\{\hat{\alpha}_{M} \pm Z_{\gamma / 2} \sqrt{\operatorname{var}\left(\hat{\alpha}_{M}\right)}\right\}, \hat{\lambda}_{M} \pm Z_{\gamma / 2} \sqrt{\operatorname{var}\left(\hat{\lambda}_{M}\right)}$ and $\left\{\hat{\beta}_{M} \pm Z_{\gamma / 2} \sqrt{\operatorname{var}\left(\hat{\beta}_{M}\right)}\right\}$ respectively, where $Z_{\gamma / 2}$ is the upper $\gamma / 2$ quantile of the standard normal distribution. Because $\alpha, \lambda$ and $\beta$ are positive parameters, it is possible to use log transformations (LT) to obtain approximate confidence intervals for these parameters (see, for example, Meeker and 
Escobar 1998 and Chan et al. 2015) by approximating the distribution of $\left(\frac{\ln (\hat{\theta})-\ln (\theta)}{\sqrt{\operatorname{var}(\ln (\hat{\theta}))}}\right)$ using a standard normal distribution, where $\operatorname{var}(\ln (\hat{\theta}))=\operatorname{var}(\hat{\theta}) / \hat{\theta}^{2}$ is an approximation of the variance of $\ln (\hat{\theta})$. Hence, the $100(1-\gamma) \%$ CIs using LT for $\alpha, \lambda$ and $\beta$ obtained in this manner are then given, respectively, by

$$
\begin{gathered}
{\left[\hat{\alpha}_{M} \exp \left(-Z_{(1-\gamma / 2)} \sqrt{\operatorname{var}\left(\ln \left(\hat{\alpha}_{M}\right)\right)} / \hat{\alpha}_{M}\right), \hat{\alpha}_{M} \exp \left(Z_{(1-\gamma / 2)} \sqrt{\operatorname{var}\left(\ln \left(\hat{\alpha}_{M}\right)\right)} / \hat{\alpha}_{M}\right)\right],} \\
{\left[\hat{\lambda}_{M} \exp \left(-Z_{(1-\gamma / 2)} \sqrt{\operatorname{var}\left(\ln \left(\hat{\lambda}_{M}\right)\right)} / \hat{\lambda}_{M}\right), \hat{\lambda}_{M} \exp \left(Z_{(1-\gamma / 2)} \sqrt{\operatorname{var}\left(\ln \left(\hat{\lambda}_{M}\right)\right)} / \hat{\lambda}_{M}\right)\right],}
\end{gathered}
$$

and

$$
\left[\hat{\beta}_{M} \exp \left(-Z_{(1-\gamma / 2)} \sqrt{\operatorname{var}\left(\ln \left(\hat{\beta}_{M}\right)\right)} / \hat{\beta}_{M}\right), \hat{\beta}_{M} \exp \left(Z_{(1-\gamma / 2)} \sqrt{\operatorname{var}\left(\ln \left(\hat{\beta}_{M}\right)\right)} / \hat{\beta}_{M}\right)\right]
$$

\subsection{Least square estimation}

In this subsection we will discuss the LS method for estimating $\alpha, \lambda$ and $\beta$. The LS estimates can be used as an input to the Newton-Raphson method to obtain the ML estimates. Let $x_{1}, x_{2}, \ldots, x_{m}$ be a progressively type ii censored sample with sample size $n$, failure information $m$ and censoring scheme $R_{1}, R_{2}, \ldots, R_{m}$ from $\operatorname{ENH}(\alpha, \lambda, \beta)$ distribution. The LS estimates can be obtained by minimizing the squared distance between the nonparametric estimates of the cdf and the parametric cdf with respect to the unknown parameters $\alpha, \lambda$ and $\beta$. Specifically, the function to be minimized is

$$
Q(\alpha, \beta, \lambda)=\sum_{i=1}^{m}\left(F\left(x_{i}\right)-\hat{F}\left(x_{i}\right)\right)^{2},
$$

where $\hat{F}\left(x_{i}\right)$ can be any nonparametric estimate of the cdf. With the progressive type-II censoring experimental scheme, the estimate of $F\left(x_{i}\right), i=1,2, \ldots, m$ is given by (Balakrishnan and Aggarwala $(2000))$

$$
\hat{F}\left(x_{i}\right)=1-\prod_{j=m-i+1}^{m} \frac{j+\sum_{k=m-j+1}^{m} R_{k}}{j+1+\sum_{k=m-j+1}^{m} R_{k}}, i=1,2, \ldots, m
$$

For the parametric cdf, Balakrishnan and Aggarwala (2000) propose

$$
F\left(x_{i}\right)=1-C_{i-1} \sum_{j=1}^{i} \frac{a_{i, j}}{\gamma_{j}}\left(1-F\left(x_{i}\right)\right)^{\gamma_{j}}
$$

where

$$
C_{i-1}=\prod_{k=1}^{i} \gamma_{k}, \gamma_{j}=m-j+1+\sum_{k=j}^{m} R_{k}, a_{i, j}=\prod_{k=1 k \neq j}^{i} \frac{1}{\gamma_{k}-\gamma_{j}}, 1 \leq j \leq i \leq m .
$$


To minimize Equation (13) with respect to $\alpha, \lambda$ and $\beta$, we differentiate with respect to these parameters, which leads to the following equations:

$$
\begin{aligned}
& 2 \beta \sum_{i=1}^{m} C_{i-1}\left[\prod_{j=m-i+1}^{m} \frac{j+\sum_{k=m-j+1}^{m} R_{k}}{j+1+\sum_{k=m-j+1}^{m} R_{k}}-C_{i-1} \sum_{j=1}^{i} \frac{a_{i, j}}{\gamma_{j}}\left[1-\left(1-e^{1-\left(1+\lambda x_{i}\right)^{\alpha}}\right)^{\beta}\right]^{\gamma_{j}}\right]\left(1+\lambda x_{i}\right)^{\alpha} \\
& \times\left(1-e^{1-\left(1+\lambda x_{i}\right)^{\alpha}}\right)^{\beta-1} \sum_{j=1}^{i} a_{i, j}\left[1-\left(1-e^{1-\left(1+\lambda x_{i}\right)^{\alpha}}\right)^{\beta}\right]^{\gamma_{i}-1} e^{1-\left(1+\lambda x_{i}\right)^{\alpha}} \log \left(1+\lambda x_{i}\right)=0 \\
& 2 \alpha \beta \sum_{i=1}^{m} C_{i-1}\left[\prod_{j=m-i+1}^{m} \frac{j+\sum_{k=m-j+1}^{m} R_{k}}{j+\sum_{k=m-j+1}^{m} R_{k}}-C_{i-1} \sum_{j=1}^{i} \frac{a_{i, j}}{\gamma_{j}}\left[1-\left(1-e^{1-\left(1+\lambda x_{i}\right)^{\alpha}}\right)^{\beta}\right]\right. \\
& \times\left(1-e^{1-\left(1+\lambda x_{i}\right)^{\alpha}}\right)^{\beta-1} \sum_{j=1}^{i} a_{i, j}\left[1-\left(1-e^{1-\left(1+\lambda x_{i}\right)^{\alpha}}\right)^{\beta} x^{\gamma_{j}-1} e^{1-\left(1+\lambda x_{i}\right)^{\alpha}}\left(1+\lambda x_{i}\right)^{\alpha-1}=0,(6)\right.
\end{aligned}
$$

and

$$
\begin{aligned}
& 2 \sum_{i=1}^{m} C_{i-1}\left[\prod_{j=m-i+1}^{m} \frac{j+\sum_{k=m-j+1}^{m} R_{k}}{j+1+\sum_{k=m-j+1}^{m} R_{k}}-C_{i-1} \sum_{j=1}^{i} \frac{a_{i, j}}{\gamma_{j}}\left[1-\left(1-e^{1-\left(1+\lambda x_{i}\right)^{\alpha}}\right)^{\beta}\right]^{\gamma_{j}}\right] \\
& \left(1-e^{1-\left(1+\lambda x_{i}\right)^{\alpha}}\right)^{\beta} \sum_{j=1}^{i} a_{i, j}\left[1-\left(1-e^{1-\left(1+\lambda x_{i}\right)^{\alpha}}\right)^{\beta}\right]^{\gamma_{j}-1} \log \left(1-e^{1-\left(1+\lambda x_{i}\right)^{\alpha}}\right)=0 .
\end{aligned}
$$

The solution of the simultaneous Equations (17)-(19) can be evaluated numerically by some suitable iterative procedure such as Newton-Raphson method, for given the values of $(n, m, \underline{R} . \underline{x})$. The LS estimates of $\alpha, \lambda$ and $\beta$ are denoted by $\hat{\alpha}_{L S}, \hat{\lambda}_{L S}$ and $\hat{\beta}_{L S}$ respectively.

\section{BOOTSTRAP CONFIDENCE INTERVALS}

Confidence intervals based on the asymptotic results are expected not to perform very well for small sample size. In this subsection, we propose to use two confidence intervals based on the parametric bootstrap methods: (i) the percentile bootstrap method (we call it Boot- $p$ ) based on the idea of Efron (1992), and (ii) the bootstrap-t method (we refer to it as Boot- $t$ ) based on the idea of Hall (1992). Due to the simplicity of the Boot-p method, it is widely used in practice. However, when the sample size is small, the percentile approach is generally not as accurate as the Boot-t approach. Below, we illustrate the steps required to estimate confidence intervals using both the methods. 


\subsection{Percentile Bootstrap (Boot-p) Confidence Interval}

Step 1 : Determine $\hat{\theta}=\left(\hat{\alpha}_{M}, \hat{\lambda}_{M}, \hat{\beta}_{M}\right)$ from the original data $x_{1}, x_{2}, \ldots, x_{m}$, using (7)-(9).

Step 2: Generate a bootstrap sample of size $m, x_{1}^{*}, x_{2}^{*}, \ldots, x_{m}^{*}$,

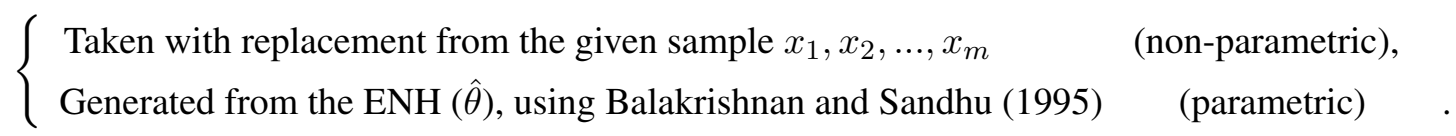

Step 3 : Based on the bootstrap sample $x_{1}^{*}, x_{2}^{*}, \ldots, x_{m}^{*}$, compute the MLE $\hat{\theta}^{*}$ of $\theta$, using (7)-(9).

Step 4 : Repeat Step 1, $B$ times to obtain a set of bootstrap samples of $\theta$, say $\left\{\hat{\theta}_{j}^{*},, j=1,2, \ldots, B\right\}$. The ordered $\hat{\theta}_{j}^{*}$ for $j=1,2, \ldots, B$ will be denoted as: $\hat{\theta}_{(1)}^{*}<\hat{\theta}_{(2)}^{*}<\ldots<\hat{\theta}_{(B)}^{*}$,

Step 5 : Let $h_{1}(y)=P\left(\hat{\theta}^{*} \leq y\right)$ be the cumulative distribution function of $\hat{\theta}^{*}$. Define $\hat{\theta}_{\mathrm{BP}}=$ $h_{1}^{-1}(y)$ for a given $y, 0<y<1$. Then the $100(1-\gamma) \%$ percentile confidence interval of $\theta=(\alpha, \lambda, \beta)$ is

$$
\left(\hat{\theta}_{\mathrm{BP}}[(\gamma / 2) B], \hat{\theta}_{\mathrm{BP}}[(1-\gamma / 2) B]\right)
$$

where, $[x]$ is the integer part of $x$.

\subsection{Student's t Bootstrap (Boot-t) Confidence Interval}

Step 1 : To obtain the Boot- $t$ confidence interval for $\theta=(\alpha, \lambda, \beta)$, we use the following algorithm:

Step 2 : From the sample $x_{1}, x_{2}, \ldots, x_{m}$ compute $\hat{\theta}=\left(\hat{\alpha}_{M}, \hat{\lambda}_{M}, \hat{\beta}_{M}\right)$.

Step 3 : Same as in Boot-p method, first generate bootstrap sample $x_{1}^{*}, x_{2}^{*}, \ldots, x_{m}^{*}$ (parametric or non-parametric), compute the MLE $\hat{\theta}^{*}=\left(\hat{\alpha}_{M}^{*}, \hat{\lambda}_{M}^{*}, \hat{\beta}_{M}^{*}\right)$ and the following statistic

$$
T^{*}=\frac{\sqrt{m}\left(\hat{\theta}^{*}-\hat{\theta}\right)}{\sqrt{\operatorname{var}\left(\hat{\theta}^{*}\right)}}
$$

where $\operatorname{var}\left(\hat{\theta}^{*}\right)$ is obtained using the observed Fisher information matrix $I^{-1}\left(\hat{\theta}^{*}\right)$, given by (12).

Step 4 : Repeat 1 and $2 B$ times and obtain $T_{(1)}^{*}, T_{(2)}^{*}, \ldots, T_{(B)}^{*}$.

Step 5 : Let $h_{2}(y)=P\left(T^{*} \leq y\right)$ be the cumulative distribution function of $T^{*}$. For a given $y$, define $\hat{\theta}_{\mathrm{Bt}}=\hat{\theta}+h_{2}^{-1}(y) \sqrt{\frac{\operatorname{var}(\hat{\theta})}{m}}$. Then the $100(1-\gamma) \%$ percentile confidence interval of $\theta=(\alpha, \lambda, \beta)$ is

$$
\left(\hat{\theta}_{\mathrm{Bt}}[(\gamma B / 2)], \hat{\theta}_{\mathrm{Bt}}[(1-\gamma / 2)] B\right) .
$$




\section{BAYESIAN ESTIMATION}

The Bayesian procedure is an attractive inferential method for small sample sizes and censored sample problems when some prior information on the parameters of the distribution are available. In this section, we propose the Bayesian approach to infer the parameter estimation of ENH distribution based on observed progressive type-II censoring.

Under the Bayes framework, it is assumed that there are prior information about the parameters that we are interested in. The prior information is often given in the form of a prior distribution. The prior distribution may reflect a strong belief so that a parameter is well-centered with a narrow range of variation (informative prior) or lack of specific knowledge so a non-informative prior is used. Bayesians often use the Gamma as a prior distribution for parameters that must be positive. The choice of gamma priors is due to their flexibilities. They accommodate a variety of shapes depending on hyper-parameters and such prior distributions are quite flexible in nature. It is also converted as non-informative prior by setting the value of hyper-parameters are equal to zero. Also, Bayesians choose independent prior distributions, to keep the form of the posterior distribution simple. Upon observing the data, a posterior distribution is obtained combing the data with the prior to update the parameter with newly obtained information. Assuming that $\alpha, \lambda$ and $\beta$ have the following gamma prior distributions:

$$
\begin{aligned}
& \pi_{1}(\alpha) \propto \alpha^{a_{1}-1} \exp \left(-b_{1} \alpha\right), \alpha>0, \\
& \pi_{2}(\lambda) \propto \lambda^{a_{2}-1} \exp \left(-b_{2} \lambda\right), \lambda>0,
\end{aligned}
$$

and

$$
\pi_{3}(\beta) \propto \beta^{a_{3}-1} \exp \left(-b_{3} \beta\right), \beta>0
$$

Here all the hyperparameters $a_{1}, a_{2}, a_{3}, b_{1}, b_{2}$, and $b_{3}$ are known and non-negative, chosen to reflect prior knowledge about the unknown parameters $\alpha, \lambda$ and $\beta$.

Combining the prior distributions in expressions (23)-(25) with the likelihood function in expression (5) we get the following joint posterior distribution

$$
\pi(\alpha, \lambda, \beta \mid \underline{x})=\frac{\pi_{1}(\alpha) \pi_{2}(\lambda) \pi_{3}(\beta) L(\alpha, \lambda, \beta \mid \underline{x})}{\int_{0}^{\infty} \int_{0}^{\infty} \int_{0}^{\infty} \pi_{1}(\alpha) \pi_{2}(\lambda) \pi_{3}(\beta) L(\alpha, \lambda, \beta \mid \underline{x}) d \alpha d \lambda d \beta} .
$$

The denominator in Equation (26) is a normalizing constant, we see the need to calculate integrals. Moreover, we usually need to integrate out the nuisance parameters and calculate the posterior mean leading to more integration. For computation of above expression, one way would be to use numerical 
techniques. An alternative method would be to use approximation techniques. Historically, there have been a number of important techniques to do numerical integration such as Simpson's rule, the midpoint rule, and Gaussian quadrature. Moreover, modern techniques like Laplace approximation and Monte Carlo integration are available and still very important. The main focus of modern Bayesian computing, however, has been on Markov chain Monte Carlo (MCMC) methods whereby a dependent sequence of random variables are obtained with the property that in the limit these random variables have the posterior distribution. The end of these sequences can be used as a sample to approximate the posterior density via a histogram or kernel density estimator. Moreover, the posterior mean and variance can be estimated by the sample mean and variance, and posterior quantiles are estimated by sample quantiles. In fact, in many ways it is simpler to have a sample from the posterior than it is to have a complex density function. There are several different MCMC algorithms that can be used to obtain the sample draws. These include the Metropolis-Hastings (M-H) algorithm by Hastings (1970) and the Gibbs sampler algorithm described by Geman and Geman (1984) and Gelfand and Smith (1990). In the present context, the Gibbs sampler along with the M-H algorithm can be used to draw samples.

Once the posterior has been obtained, a loss function is attached to indicate the loss coming up when the estimate $\hat{\theta}$ deviates from the true value. The loss should be zero if and only if $\hat{\theta}=\theta$. The most common symmetric loss function used to measure estimator performance in practice is squared error (SE) loss function, for the reason that when using SE loss function, it gives equal weighted to overestimation as well as underestimation. If $\theta$ is the parameter to be estimated by an estimator $\hat{\theta}$, then the square error loss function is defined as

$$
L(\theta, \hat{\theta})=(\theta-\hat{\theta})^{2} .
$$

Therefore, the Bayes estimate of any function of $\alpha, \lambda$ and $\beta$, say $g(\alpha, \lambda, \beta)$ under the SE loss function (27) can be obtained as

$$
\hat{g}_{B S}(\alpha, \lambda, \beta \mid \underline{x})=E_{\alpha, \lambda \mid \underline{x}}(g(\alpha, \lambda, \beta)),
$$

where

$$
E_{\alpha, \lambda, \beta \mid \underline{x}}(g(\alpha, \lambda, \beta))=\frac{\int_{0}^{\infty} \int_{0}^{\infty} \int_{0}^{\infty} g(\alpha, \lambda, \beta) \pi_{1}(\alpha) \pi_{2}(\lambda) \pi_{3}(\beta) L(\alpha, \lambda, \beta \mid \underline{x}) d \alpha d \lambda d \beta}{\int_{0}^{\infty} \int_{0}^{\infty} \int_{0}^{\infty} \pi_{1}(\alpha) \pi_{2}(\lambda) \pi_{3}(\beta) L(\alpha, \lambda, \beta \mid \underline{x}) d \alpha d \lambda d \beta} .
$$

However, in practical situation the real loss is often not symmetric i.e., overestimation of a parameter may lead to harder (weaker) consequences than under estimation and vice-a-versa. Therefore, in order to cope up with such situations, the use of asymmetric loss function has been suggested by many 
authors (Zellner, 1986; Varian, 1975; Berger, 1985). There are several asymmetric loss functions proposed, and one of the most popular asymmetric loss function is the linear-exponential (LINEX) loss function, $L(\Delta)$, introduced by Varian (1975), which is defined as

$$
L(\Delta)=\left(e^{c \Delta}-c \Delta-1\right), c \neq 0, \Delta=\hat{\theta}-\theta,
$$

where $c$ is a scale parameter of the loss function. For small values of $c$ (near to zero), the LINEX loss function is almost the same as the SE loss function and for the choice of negative or positive values of $c$, the LINEX loss function gives more weight to overestimation or underestimation (for details, see Zellner (1986)). This loss function is suitable for situations where overestimation of is more costly than its underestimation. Under LINEX loss function in (30), the Bayes estimate of a function $g(\alpha, \lambda, \beta)$ is

$$
\hat{g}_{B L}(\alpha, \lambda, \beta \mid \underline{x})=\frac{-1}{c} \log \left[E\left(e^{-c g(\alpha, \lambda, \beta)} \mid \underline{x}\right)\right], c \neq 0,
$$

where

$$
E\left(e^{-c g(\alpha, \lambda, \beta)} \mid \underline{x}\right)=\frac{\int_{0}^{\infty} \int_{0}^{\infty} \int_{0}^{\infty} e^{-c g(\alpha, \lambda, \beta)} \pi_{1}(\alpha) \pi_{2}(\lambda) \pi_{3}(\beta) L(\alpha, \lambda, \beta \mid \underline{x}) d \alpha d \lambda d \beta}{\int_{0}^{\infty} \int_{0}^{\infty} \int_{0}^{\infty} \pi_{1}(\alpha) \pi_{2}(\lambda) \pi_{3}(\beta) L(\alpha, \lambda, \beta \mid \underline{x}) d \alpha d \lambda d \beta}
$$

Furthermore; there is another loss function, called general entropy (GE) loss function, proposed by Calabria and Pulcini (1994), which is given by,

$$
L(\hat{\theta}-\theta) \propto\left(\frac{\hat{\theta}}{\theta}\right)^{q}-q \ln \left(\frac{\hat{\theta}}{\theta}\right)-1
$$

This loss function is a generalization of the entropy loss function used by several authors where the shape parameter $q$ is taken equal to 1 . For $q=-1$ the GE loss function simplifies to the SE loss function and the weighted squared-error loss function results from $q=1$. For $q=-2$, the general entropy loss function is referred to as the precautionary loss function. The general version (33) allows different shapes of the loss function to meet the practical needs. It may be noted that when $q>0$, a positive error causes more serious consequence than a negative error and reverse is the situation when $q<0$. Under the general entropy loss (33), the Bayes estimator of $g(\alpha, \lambda, \beta)$ is then given by

$$
\left.\hat{g}_{B G E}(\alpha, \lambda, \beta \mid \underline{x})=\left[E(g(\alpha, \lambda, \beta))^{-q} \mid \underline{x}\right)\right]^{\frac{-1}{q}},
$$

where

$$
E\left(g(\alpha, \lambda, \beta)^{-q} \mid \underline{x}\right)=\frac{\int_{0}^{\infty} \int_{0}^{\infty} \int_{0}^{\infty} g(\alpha, \lambda, \beta)^{-q} \pi_{1}(\alpha) \pi_{2}(\lambda) \pi_{3}(\beta) L(\alpha, \lambda, \beta \mid \underline{x}) d \alpha d \lambda d \beta}{\int_{0}^{\infty} \int_{0}^{\infty} \int_{0}^{\infty} \pi_{1}(\alpha) \pi_{2}(\lambda) \pi_{3}(\beta) L(\alpha, \lambda, \beta \mid \underline{x}) d \alpha d \lambda d \beta}
$$


It should be mentioned the above expressions $(29,32,35)$ cannot be obtained in nice closed form, this is due to the complex form of the likelihood function given in (5). We derive the Gibbs sampling technique to obtain the Bayesian estimators and the $95 \%$ CIs of $\alpha, \lambda$ and $\beta$.

The joint posterior (26) can be written in the following form

$$
\begin{aligned}
p(\alpha, \lambda, \beta \mid \underline{x}) \propto & \alpha^{m+a_{1}-1} \lambda^{m+a_{2}-1} \beta^{m+a_{3}-1} e^{-\sum_{i=1}^{m}\left(1+\lambda x_{i}\right)^{\alpha}+b_{1} \alpha+b_{2} \lambda+b_{3} \beta} \prod_{i=1}^{m} \frac{\left(1+\lambda x_{i}\right)^{\alpha-1}}{\left(1-e^{\left.1-\left(1+\lambda x_{i}\right)^{\alpha}\right)}\right.} \\
& \times \prod_{i=1}^{m} \sum_{k=0}^{\infty}\left(\begin{array}{c}
R_{i} \\
k
\end{array}\right)(-1)^{k}\left(1-e^{1-\left(1+\lambda x_{i}\right)^{\alpha}}\right)^{\beta(k+1)} .
\end{aligned}
$$

The full conditional posterior densities of $\alpha, \lambda$ and $\beta$ obtained from (36), are given below

$$
\begin{aligned}
p_{1}(\alpha \mid \underline{x}, \lambda, \beta) \propto & \alpha^{m+a_{1}-1} e^{-b_{1} \alpha+\sum_{i=1}^{m}\left(1+\lambda x_{i}\right)^{\alpha}} \prod_{i=1}^{m} \frac{\left(1+\lambda x_{i}\right)^{\alpha}}{\left(1-e^{1-\left(1+\lambda x_{i}\right)^{\alpha}}\right)} \\
& \times \prod_{i=1}^{m} \sum_{k=0}^{\infty}\left(\begin{array}{c}
R_{i} \\
k
\end{array}\right)(-1)^{k}\left(1-e^{1-\left(1+\lambda x_{i}\right)^{\alpha}}\right)^{\beta(k+1)}, \\
p_{2}(\lambda \mid \underline{x}, \alpha, \beta) \propto & \lambda^{m+a_{2}-1} e^{-b_{2} \lambda+\sum_{i=1}^{m}\left(1+\lambda x_{i}\right)^{\alpha}} \prod_{i=1}^{m} \frac{\left(1+\lambda x_{i}\right)^{\alpha-1}}{\left(1-e^{\left.1-\left(1+\lambda x_{i}\right)^{\alpha}\right)}\right.} \\
& \times \prod_{i=1}^{m} \sum_{k=0}^{\infty}\left(\begin{array}{c}
R_{i} \\
k
\end{array}\right)(-1)^{k}\left(1-e^{1-\left(1+\lambda x_{i}\right)^{\alpha}}\right)^{\beta(k+1)},
\end{aligned}
$$

and

$$
p_{3}(\beta \mid \underline{x}, \alpha, \lambda) \propto \beta^{m+a_{3}-1} e^{-b_{3} \beta} \prod_{i=1}^{m} \sum_{k=0}^{\infty}\left(\begin{array}{c}
R_{i} \\
k
\end{array}\right)(-1)^{k}\left(1-e^{1-\left(1+\lambda x_{i}\right)^{\alpha}}\right)^{\beta(k+1)} .
$$

Clearly, the form of the conditional distributions (37)-(39) do not belong to any known parametric distribution family, therefore we propose the implementation of $\mathrm{M}-\mathrm{H}$ algorithm with Gibbs iterations and hence, the following algorithm is proposed to obtain the MCMC samples and then to construct the Bayesian estimators of $\alpha, \lambda$ and $\beta$ and the CIs:

Step 1 : Start with initial guess of $\alpha, \lambda$ and $\beta$, say $\alpha^{(0)}, \lambda^{(0)}$ and $\beta^{(0)}$. We use the ML estimates for these initial values.

Step 2 : Set $j=1$.

Step 3 : Generate $\alpha^{(j)}$ from $p_{1}\left(\alpha \mid \underline{x}, \lambda^{(j-1)}, \beta^{(j-1)}\right)$ with normal proposal distribution, $N\left(\alpha^{(j-1)}, V_{\alpha}\right)$, using the following Metropolis-Hastings: 
(i) Generate a proposal $\alpha^{*}$ from $N\left(\alpha^{(j-1)}, V_{\alpha}\right)$.

(ii) Evaluate the acceptance probabilities $\rho_{\alpha}=\min \left[1, \frac{p_{1}\left(\alpha^{*} \mid \underline{x}, \lambda^{(j-1)}, \beta^{(j-1)}\right)}{p_{1}\left(\alpha^{(j-1)} \mid \underline{x}, \lambda^{(j-1)}, \beta^{(j-1)}\right)}\right]$.

(iii) Generate a $u_{1}$ from a uniform $(0,1)$ distribution.

(iv) If $u_{1} \leq \rho_{\alpha}$, accept the proposal and set $\alpha^{(j)}=\alpha^{*}$, eLS estimates set $\alpha^{(j)}=\alpha^{(j-1)}$.

Step 4 : Like the previous step, generate $\lambda^{(j)}$ and $\beta^{(j)}$ from $p_{2}\left(\lambda \mid \underline{x}, \alpha^{(j)}, \beta^{(j-1)}\right)$ and $p_{3}\left(\beta \mid \underline{x}, \alpha^{(j)}, \lambda^{(j)}\right)$ with normal proposal distributions, $N\left(\lambda^{(j-1)}, V_{\lambda}\right)$ and $N\left(\beta^{(j-1)}, V_{\beta}\right)$.

Step 5 : Repeat Steps 2-3, $N$ times. In practice, one typically generates a sequence of length $N$ but discards the first $M$ burn-in elements as a means of assuring convergence to the stationary distribution of the Markov chain. Then, the remaining $N-M$ elements of the sequence are used to estimate functions of the posterior distribution such as the mean, standard deviation, quantiles, etc.

Step 6 : Obtain the Bayes estimate of $\theta=(\alpha, \lambda$ or $\beta)$, under SE, LINEX and GE loss functions, respectively, as

$$
\begin{aligned}
& \hat{\theta}_{B S}=\frac{1}{N-M} \sum_{j=M+1}^{N} \theta^{(j)}, \\
& \hat{\theta}_{B l}=\frac{-1}{c} \log \left[\frac{1}{N-M} \sum_{j=M+1}^{N} e^{-c \theta^{(j)}}\right],
\end{aligned}
$$

and

$$
\hat{\theta}_{B G E}=\left[\frac{1}{N-M} \sum_{j=M+1}^{N}\left(\theta^{(j)}\right)^{-q}\right]^{\frac{-1}{q}}
$$

Step 7 : To compute the credible intervals of $\theta=(\alpha, \lambda$ or $\beta)$, order $\theta^{(j)}, j=1,2, \ldots, N$, as $\theta_{(1)}<\theta_{(1)}<\ldots<\theta_{(N)}$. Then, the $100(1-\gamma) \%$ Bayesian credible intervals of $\theta$ become $\left(\theta_{(N \gamma / 2)}, \theta_{(N(1-\gamma / 2))}\right)$.

\section{Application of ENH Distribution to LifETime Data}

The body is made up of trillions of living cells. Normal body cells grow, divide to make new cells, and die in an orderly way. During the early years of a person's life, normal cells divide faster to allow the person to grow. After the person becomes an adult, most cells divide only to replace worn-out or dying cells or to repair injuries. Cancer begins when cells in a part of the body start to grow out of control. There are many kinds of cancer, but they all start because of out-of-control growth of 
abnormal cells. Different types of cancer can behave very differently. They grow at different rates and respond to different treatments. Bladder cancer is a disease in which malignant (cancer) cells form in the tissues of the bladder. It is one of the most expensive cancers for society because patients live longer and have multiple recurrences.

In this section an uncensored data set corresponding to remission times (in months) of a random sample of 128 bladder cancer patients is considered. These data were previously studied by Lee and Wang (2003), Lemonte and Cordeiro (2011) and Lemonte (2013). The remission times of the bladder cancer are as follows:

$x^{(0)}=(0.08,0.20,0.40,0.50,0.51,0.81,0.90,1.05,1.19,1.26,1.35,1.40,1.46,1.76,2.02,2.02$, $2.07,2.09,2.23,2.26,2.46,2.54,2.62,2.64,2.69,2.69,2.75,2.83,2.87,3.02,3.25,3.31,3.36,3.36$, $3.48,3.52$, 3.57, 3.64, 3.70, 3.82, 3.88, 4.18, 4.23, 4.26, 4.33, 4.34, 4.40, 4.50, 4.51, 4.87, 4.98, 5.06, 5.09, 5.17, 5.32, 5.32, 5.34, 5.41, 5.41, 5.49, 5.62, 5.71, 5.85, 6.25, 6.54, 6.76, 6.93, 6.94, 6.97, 7.09, 7.26, 7.28, 7.32, 7.39, 7.59, 7.62, 7.63, 7.66, 7.87, 7.93, 8.26, 8.37, 8.53, 8.65, 8.66, 9.02, 9.22, 9.47, $9.74,10.06,10.34,10.66,10.75,11.25,11.64,11.79,11.98,12.02,12.03,12.07,12.63,13.11,13.29$, $13.80,14.24,14.76,14.77,14.83,15.96,16.62,17.12,17.14,17.36,18.10,19.13,20.28,21.73$, $22.69,23.63,25.74,25.82,26.31,32.15,34.26,36.66,43.01,46.12,79.05)$.

These remission times are a subset of the bladder cancer data and are used here only for illustrative purposes. Lemonte (2013) reported that the $\operatorname{ENH}(\alpha, \lambda, \beta)$ distribution fits the data quite well.

First, we compute the ML, LS and Bayes estimators of the parameters $\alpha, \beta$ and $\lambda$ based on the complete data. The results of ML and LS estimates are presented in Table 1. Based on the ML estimates of $\alpha, \lambda$ and $\beta$, the observed variance-covariance matrix is

$$
\left(\begin{array}{ccc}
0.0137671 & -0.0197632 & -0.0322625 \\
-0.0197632 & 0.0307219 & 0.0554005 \\
-0.0322625 & 0.0554005 & 0.1329940
\end{array}\right)
$$

The $95 \%$ confidence intervals of $\alpha, \lambda$ and $\beta$ based on ML estimates, obtained by AN or LT technique, and parametric bootstrap, as suggested in Section 2 and Section 3, are obtained and the results are reported in Table 2 . In the absence of any strong prior information, we use noninformative prior distributions for $\alpha, \beta$ and $\lambda$, i.e. $a_{1}=b_{1}=a_{2}=b_{2}=a_{3}=b_{3}=0$. Then the joint posterior density is proportional to the likelihood function. The Bayes estimators cannot be found in closed form. Thus, we must resort to using a Markov Chain Monte Carlo (MCMC) method to compute Bayes estimators. We implement the Gibbs with in $\mathrm{M}-\mathrm{H}$ algorithm to sample from the posterior distribution of $\alpha, \beta$ and $\lambda$. We run the chain for 30000 times and discard the first 5000 values as burn in. The 
burn in is needed because of the fact that, because chains are initialized with values not actually drawn from the posterior distribution, the simulated values of $\alpha, \beta$ and $\lambda$ obtained at the beginning of a MCMC run are not distributed from the posterior distributions. However, after some number of iterations have been performed (the burn-in period), the effect of the initial values wears off and the distribution of the new iterates approaches the true posterior distribution. The Bayes estimate of $\alpha$, $\lambda$ and $\beta$ are found also in Table 1. Trace plot and corresponding histogram of 25000 iterations relate to $x^{(0)}$ are listed in Figures. 1 and 2, respectively. From Figures. 1 and 2, we can see that the Gibbs with in M-H sampling is convergent and the densities of $\alpha, \lambda$ and $\beta$ are almost symmetric. Further; the $95 \%$ Bayesian credible intervals are tabulated in Table 2.

Table 1: K-S distances and the corresponding $p$-values for ML, LS and Bayes estimates.

\begin{tabular}{ccccccc}
\hline Method & Data & $\alpha$ & $\lambda$ & $\beta$ & K-S & $p$ value \\
\hline ML & $x^{(0)}$ & 0.6372 & 0.3444 & 1.6884 & 0.0442 & 0.9639 \\
& $x^{(1)}$ & 0.7997 & 0.1834 & 1.4573 & 0.0711 & 0.7653 \\
\hline LS & $x^{(0)}$ & 0.6344 & 0.3457 & 1.6944 & 0.0428 & 0.9731 \\
& $x^{(1)}$ & 1.2685 & 0.0917 & 1.4103 & 0.0715 & 0.7592 \\
\hline \multirow{2}{*}{ Bayes (SE) } & $x^{(0)}$ & 0.6373 & 0.3450 & 1.6970 & 0.0431 & 0.9713 \\
& $x^{(1)}$ & 0.6388 & 0.3089 & 1.6849 & 0.0697 & 0.7860 \\
\hline
\end{tabular}

Table 2: The 95\% CIs of $\alpha, \lambda$ and $\beta$ based on ML, Bayesian and bootstrapping methods.

\begin{tabular}{cc|c|c|c}
\hline Method & Data & $\alpha$ & $\lambda$ & $\beta$ \\
\hline AN & $x^{(0)}$ & $(0.4072,0.8672) 0.4600$ & $(0.0009,0.6880) 0.6871$ & $(0.9736,2.4032) 1.4296$ \\
& $x^{(1)}$ & $(0.4446,1.1547) 0.7102$ & $(0.0018,0.3649) 0.3631$ & $(0.9168,1.9979) 1.0811$ \\
\hline LT & & $(0.4442,0.9142) 0.4700$ & $(0.1270,0.9338) 0.8068$ & $(1.1057,2.5783) 1.4726$ \\
\hline & & $(0.5129,1.2467) 0.7337$ & $(0.0681,0.4936) 0.4254$ & $(1.0057,2.1118) 1.1060$ \\
\hline Bayes & $x^{(0)}$ & $(0.6260,0.6484) 0.0224$ & $(0.2550,0.4453) 0.1903$ & $(1.2761,2.2078) 0.9317$ \\
& $x^{(1)}$ & $(0.6198,0.6555) 0.0356$ & $(0.2138,0.4007) 0.1874$ & $(1.2068,2.1831) 0.9762$ \\
\hline Boot- $p$ & $x^{(0)}$ & $(0.5408,0.7391) 0.1983$ & $(0.2798,0.6325) 0.3527$ & $(1.4235,2.4126) 0.9891$ \\
& $x^{(1)}$ & $(0.7446,1.9047) 1.1601$ & $(0.0525,0.2138) 0.1613$ & $(1.1008,1.8738) 1.7730$ \\
\hline Boot- $t$ & $x^{(0)}$ & $(0.4865,0.8928) 0.4063$ & $(0.1702,0.7109) 0.5407$ & $(1.1542,2.1754) 1.0212$ \\
& $x^{(1)}$ & $(0.5288,1.0815) 0.5527$ & $(0.1054,0.5337) 0.4283$ & $(1.2590,2.5518) 1.2928$ \\
\hline
\end{tabular}

Furthermore, it is well known for the LINEX loss function that $c>0$ implies that overestimation results is more penalty than underestimation and reverse is true for $c<0$. Also LINEX loss function becomes symmetric for $c$ close to zero and hence approximately behaves as the SE loss function 
itself. In a similar manner it is to be noted for the loss function GE that $q>0$ means overestimation is more serious than under estimation and opposite is true for $q<0$. The case $q=-1$ approximately corresponds to the loss function SEL. With $c=-200,0.5,200$ and $q=-200,0.5,200$, the result of the Bayes estimates of $\alpha, \lambda$ and $\beta$ under LINEX and GE loss functions, relative to $x^{(0)}$ and $x^{(1)}$ are reported in Table 3.

Table 3: Bayesian estimates of $\alpha, \lambda$ and $\beta$ under LINEX and GE.

\begin{tabular}{|c|c|c|c|c|c|c|c|}
\hline \multirow{2}{*}{ Estimate } & & \multicolumn{3}{|c|}{ LINEX } & \multicolumn{3}{c|}{ GE } \\
\cline { 3 - 8 } & Data & $c=-200$ & $c=0.5$ & $c=200$ & $q=-200$ & $q=0.5$ & $q=200$ \\
\hline$\alpha$ & $x^{(0)}$ & 0.6407 & 0.6373 & 0.6338 & 0.6425 & 0.6372 & 0.6318 \\
& $x^{(1)}$ & 0.6466 & 0.6389 & 0.6319 & 0.6410 & 0.6387 & 0.6292 \\
\hline$\lambda$ & $x^{(0)}$ & 0.4958 & 0.3444 & 0.2175 & 0.5144 & 0.3398 & 0.1855 \\
& $x^{(1)}$ & 0.4035 & 0.3083 & 0.2022 & 0.4172 & 0.3035 & 0.1776 \\
\hline$\beta$ & $x^{(0)}$ & 2.4097 & 1.6831 & 0.9855 & 2.3596 & 1.6723 & 0.9841 \\
& $x^{(1)}$ & 2.3293 & 1.6719 & 1.0722 & 2.2914 & 1.6611 & 1.0740 \\
\hline
\end{tabular}

For analyzing the data set under progressive type ii censoring scheme, we have generated some artificial progressive type ii censoring sample from $x^{(0)}$, with $m=88, R_{3}=R_{11}=R_{15}=R_{33}=$ $10, R_{i}=0$ for $i \neq 3,11,15,33$, say $x^{(1)}$ as follows:

$x^{(1)}=(0.08,0.2,0.4,0.5,0.51,0.81,0.9,1.05,1.19,1.26,1.35,1.4,2.02,2.02,2.07,2.26,2.46$, 2.64, 2.69, 2.75, 3.02, 3.31, 3.57, 3.64, 3.7, 3.82, 3.88, 4.18, 4.26, 4.33, 4.34, 4.5, 5.09, 5.17, 5.32, 5.34, 5.49, 5.62, 5.85, 6.25, 6.54, 6.93, 6.94, 7.26, 7.28, 7.32, 7.39, 7.59, 7.62, 7.63, 7.66, 7.87, 8.26, $8.53,8.65,8.66,9.02,9.22,9.47,10.34,10.66,10.75,11.25,11.64,11.79,12.02,12.03,12.07,12.63$, $13.11,13.8,14.24,14.76,14.77,14.83,15.96,16.62,17.12,17.36,19.13,20.28,22.69,23.63,25.74$, $25.82,26.31,46.12,79.05)$.

For this sample, the ML, LS and Bayes estimates of the parameters $\alpha, \lambda$ and $\beta$ are allso displayed in Table 1. Here, we also mention that all Bayes estimates are computed with respect to the noninformative prior distribution. The 95\% CIs based on ML, Bayesian, Boot- $p$ and Boot- $t$ methods are presented in Table 2. As the case of the complete sample, the Bayes estimates with resp;gect to the LINEX loss function are computed for three distinct values of $c$, namely $-0.200,0.5,200$. Also, under GE loss associated estimates are obtained for $q=-0.200,0.5,200$. The results are reported In Table 3.

To check the validity of the model, we compute the Kolmogorov-Smirnov (K-S) distance between the empirical distribution function and the fitted distribution functions and the corresponding $p$-value 
when the parameters are obtained by different methods. The results are presented also in Table 1 . From Table 1, it is observed that the estimated ENH distribution provide excellent good fit to the given data. Furthermore, from the results obtained in Tables 1, 2, we can conclude that the Bayesian procedure provides the more accurate and precise estimates of the parameters even if we consider the noninformative prior. Also, it is easy to see that the estimates of the LS and Bayesian are very close to the ML estimators that Lemonte (2013) got.
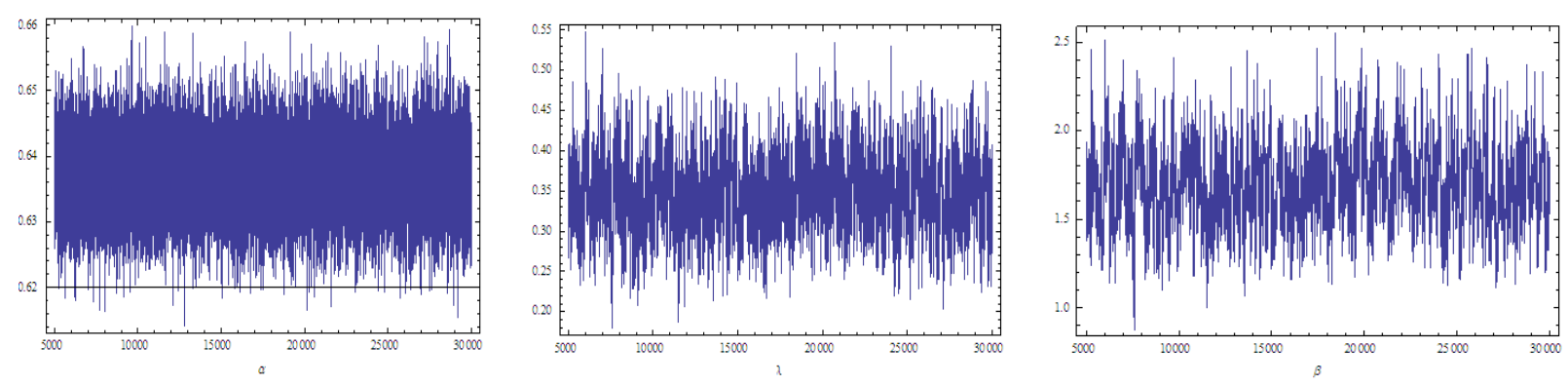

Figure 1: Trace plots of the parameters obtained from the Gibbs sampling of $x^{(0)}$
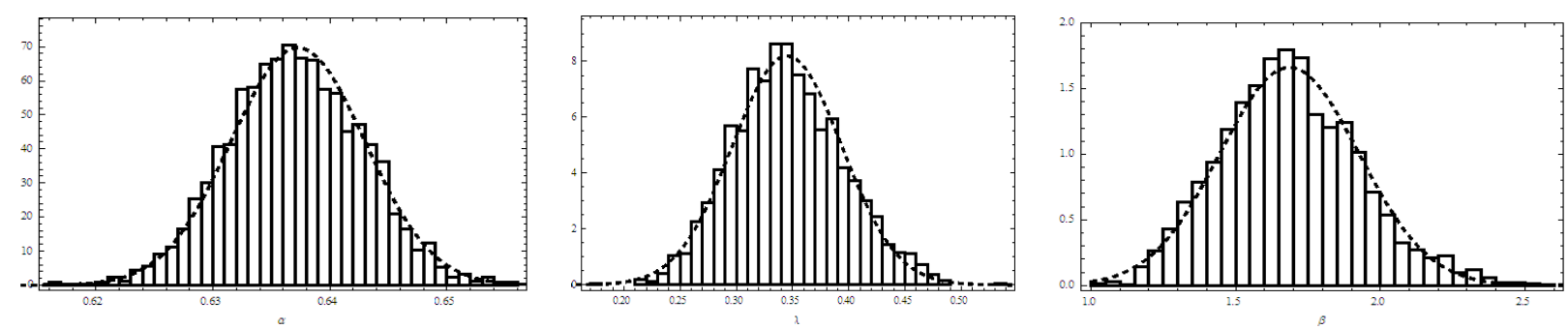

Figure 2: Histograms and approximate marginal posteriors for the parameters of $4 x^{(0)}$

\section{Simulation STUDY}

It is natural after completing theoretical calculations in previous sections, going into practical calculations. Therefore, in this section, we conduct a Monte Carlo simulation study to make a small comparison between the classical estimates (MLE and LSE) and the Bayesian estimates. We mainly compute the avrege estimates (AE), the biases and mean square errors (MSEs) of the MLEs, LSEs and Bayes estimates. Based on symmetric and asymmetric loss functions, using informative gamma prior, we conduct the Bayes estimates of $\alpha, \lambda$ and $\beta$. Here, hyperparameters are so chosen that prior 
means are exactly equal to the true values of the parameters. The true value of $(\alpha, \lambda, \beta)$ is taken as $(2.0,1.0,2.0)$ and hyperparameters are assigned values of $a_{1}=0.2, b_{1}=0.1, a_{2}=0.1, b_{2}=0.1$, $a_{3}=0.2, b_{3}=0.1$. The Bayes estimates with respect to the LINEX and GE loss functions are computed for three different values of $c$ and $q$, namely -2, 0.5, 2. For MCMC technique, we use $N=5000$ with burn-in-period $M=1000$. All the results are based on the 1000 progressively censored samples of size $m=15,20,25,30,50$ from a sample of size $n=20,25,30,50$ and various progressive-censoring schemes $R$, generated using algorithm presented by Balakrishnan and Sandhu (1995). The results of the simulation study are computed and reported in Tables 4-6. On the other hand, the asymptotic 95\% confidence intervals based on the asymptotic distributions of the MLE, Bayes credible intervals and two parametric bootstrap, namely Boot-p and Boot-t of unknown model parameters $\alpha, \lambda$ and $\beta$ are displayed in Table 7. For the bootstrap methods, we compute the confidence intervals based on $B=400$ resampling. We compare different confidence intervals in terms of average lengths and coverage probabilities. The nominal level for the confidence intervals or the credible intervals is 0.95 in each case.

Table 4-6 shows that the Bayes estimates in all considered loss functions are better than other estimates (maximum likelihood and list squared estimators) in terms of both bias and MSE values. This holds for $\alpha, \lambda$ and $\beta$ and almost for all tabulated values. Furthermore the least square method provide good estimates for the unknown parameters $\alpha, \lambda$ and $\beta$ at least in comparison with corresponding MLEs. We observed also that, in most of the cases, the bias and MSE increase as the $n$ and $m$ decreases.

From Table 7, we observe that the approximate confidence intervals based on the asymptotic distributions of the MLE are wider than the other confidence intervals. We also observed that the Bayesian credible intervals provide the smallest average confidence credible lengths for different censoring schemes. Finally, it is also observed that Boot-t confidence intervals perform better than the Boot-p confidence intervals. It is seen that, with the larger sample size average interval length decreases. It is also seen that all the three intervals show pleasurable coverage probabilities.

\section{Conclusions}

The classical and the Bayesian methods are two types of inference procedures have considered here to estimate the unknown parameters of the ENH distribution when data under consideration are progressively type-II censoring. For classical approach, the maximum likelihood (ML) as well as list square (LS) estimates do not have explicit forms. Therefore, the Newton-Raphson algorithm has been used to compute the MLEs as well as LSEs. NMaximize and NMinmize are two commands in Mathematica 
are used to give the optimum solutions of MLEs and LSEs, respectively. The use of these commands does not require initial values. For the Bayesian approach, the Bayes estimates of the unknown parameters are computed based on symmetrical (squared error) and asymmetrical (LINEX and general entropy) loss functions, using non-informative (see real example) and gamma informative priors (see simulation study). With the MCMC technique, the posterior samples are obtained to compute Bayes estimates of the unknown parameters and the associated credible intervals. Also, we have derived the MLEs and bootstrap confidence intervals of the unknown parameters. One data set and a Mont Carlo simulation study have been analyzed. It is observed that Methods of ML, LS and Bayes of the unkown parameters gives best performance for the real data. Furthermore, our simulation study reveals that Bayes estimates under different loss functions are better than all estimates used in this study.

\section{ACKNOWLEDGEMENT}

The authors would like to thank the Editor and reviewers for their valuable comments and suggestions to improve the presentation of the paper. The authors would like to thank the Deanship of Scientific Research at Majmaah University for supporting this work under Project No. 1440-142.

\section{REFERENCES}

1. E. A. Ahmed, Bayesian estimation based on progressive Type-II censoring from two-parameter bathtubshaped lifetime model: an Markov chain Monte Carlo approach, J. Appl. Stat., 41 (2014), 752-768.

2. E. A. Ahmed, Estimation of some lifetime parameters of generalized Gompertz distribution under progressively type-II censored data, Appl. Math. Modell, 39 (2015), 5567-5578.

3. N. Balakrishnan, Progressive censoring methodology: an appraisal (with discussions), Test, 16 (2007) 211-296

4. N. Balakrishnan and R. Aggarwala, Progressive censoring: theory, methods, and applications, Boston: Birkhauser. (2000).

5. N. Balakrishnan and E. Cramer, The arts of progressive censoring, Birkhauser: New York, NY, USA (2014).

6. N. Balakrishnan and R. A. Sandhu, A simple simulational algorithm for generating progressive Type-II censored samples, Amer. Statist., 49 (1995), 229-230.

7. J. O. Berger, Statistical decision theory and bayesian analysis, Springer, New York, (1985).

8. R. Calabria and G. Pulcini, An engineering approach to Bayes estimation for the Weibull distribution, Microelectronics Reliability, 34 (1994), 789-802. 
9. B. Efron, Jackknife - after - bootstrap standard errors and infl uence functions, J. R. Stat. Soc. B, 54 (1992), 83-127.

10. A. El-Gohary, A. Alshamrani, and A. Al-Otaibi, The generalized Gompertz distribution, Applied Mathematical Modeling, 37 (2013), 13-24.

11. A. E. Gelfand and A. F. M. Smith, Sampling-based approaches to calculating marginal densities, Journal of the American Statistical Association, 85 (1990), 398-409.

12. S. Geman and D. Geman, Stochastic relaxation, Gibbs distributions and the Bayesian restoration of images, IEEE Trans. Pattern Anal. Mach. Intell., 6 (1984), 721-741.

13. R.D. Gupta and D. Kundu, Generalized exponential distributions, Australian and New Zealand Journal of Statistics, 41 (1999), 173-188.

14. R. D. Gupta and D. Kundu, Exponentiated exponential family: An alternative to gamma and weibull distributions, Biometrical Journal, 43 (2001), 117-130.

15. P. Hall, The bootstrap and Edgeworth expansion, Springer- Verlag, New York, (1992).

16. U. Hjorth, A reliability distribution with increasing, decreasing, constant and bathtub-shaped failure rates, In: Technometrics, 22 (1980), 99-109.

17. C. D. Lai, M. Xie, and D. N. P. Murthy, A modified Weibull distribution, IEEE Transactions on Reliability, 52 (2003), 33-37.

18. E. T. Lee and J. W. Wang, Statistical methods for survival data analysis (3rd ed.), New York: Wiley, (2003).

19. A. J. Lemonte, A new exponential-type distribution with constant, decreasing, increasing, upside-down bathtub and bathtub-shaped failure rate function, Computational Statistics and Data Analysis, 62 (2013), 149-170.

20. A. J. Lemonte and G. M. Cordeiro, The exponentiated generalized inverse Gaussian distribution, Statistics and Probability Letters, 81 (2011), 506-517.

21. A. W. Marshall and I. Olkin, A new method for adding a parameter to a family of distributions with application to the exponential and Weibull families, Biometrika, 84 (1997), 641-652.

22. G. S. Mudholkar and D. K. Srivastava, Exponentiated Weibull family for analyzing bathtub failure-real data, IEEE Transaction Reliability, 42 (1993), 299-302.

23. S. Nadarajah and F. Haghighi, An extension of the exponential distribution, Statistics, 45 (2011), 543558.

24. S. Rajarshi and M. B. Rajarshi, Bathtub distributions: A review, Communication in Statistics - Theory Methods, 17 (1988), 2597-2621. 
25. G. O. Silva, E. M. M. Ortega, and G. M. Cordeiro, The beta modified Weibull distribution, Lifetime Data Anaysis, 16 (2010), 409-430.

26. S. K. Singh, U. Singh, and M. Kumar, Bayesian inference for exponentiated Pareto model with application to bladder cancer remission time, Transition in Statistics, 15 (2014), 403-426.

27. A. A. Soliman, A. H. Abd-Ellah, N. A. Abou-Elheggag, and E. A. Ahmed, Modified Weibull model: A Bayes study using MCMC approach based on progressive censoring data, Reliability Engineering and System Safety, 100 (2012), 48-57.

28. H. R. Varian, A Bayesian approach to real estate assessment, North Holland, Amsterdam , (1975), 195208.

29. M. Xie, Y. Tang, and T. N. Goh, A modified Weibull extension with bathtub-shaped failure rate function, Reliability Engineering and System Safety, 76 (2002), 279-285.

30. T. Zhang and M. Xie, Failure data analysis with extended Weibull distribution, Communications in Statistics-Simulation and Computation, 36 (2007), 579-592.

31. A. Zellner, Bayesian estimation and prediction using asymmetric loss functions, J. Am. Stat. Assoc., 81 (1986), 446-545. 
Table 4. Avrege estimates (AE), MSEs and baises of ML, LS and Bayes estimates under SE, LINEX and GE of $\alpha$

\begin{tabular}{|c|c|c|c|c|c|c|c|c|c|c|c|}
\hline \multirow[b]{2}{*}{$(n, m)$} & \multirow[b]{2}{*}{ Scheme } & & \multirow[t]{2}{*}{ MLE } & \multirow[t]{2}{*}{ LSE } & \multirow[t]{2}{*}{ SEL } & \multicolumn{3}{|c|}{ LINEX } & \multicolumn{3}{|c|}{ GE } \\
\hline & & & & & & $c=-2$ & $c=0.5$ & $c=2$ & $q=-2$ & $q=0.5$ & $q=2$ \\
\hline \multirow[t]{9}{*}{$(20,15)$} & $\left(5,0^{* 14}\right)$ & $\mathrm{AE}$ & 2.3278 & 2.3949 & 2.0398 & 2.2734 & 1.9861 & 1.8566 & 2.0892 & 1.9655 & 1.8932 \\
\hline & & MSE & 1.5236 & 0.5973 & 0.2280 & 0.4870 & 0.1929 & 0.1587 & 0.2556 & 0.1996 & 0.1884 \\
\hline & & BISE & 0.9685 & 0.6887 & 0.3686 & 0.4972 & 0.3468 & 0.3246 & 0.3843 & 0.3529 & 0.3497 \\
\hline & $\left(1^{* 5}, 0^{* 10}\right)$ & $\mathrm{AE}$ & 2.2167 & 2.3834 & 2.0473 & 2.2701 & 1.9954 & 1.8661 & 2.0950 & 1.9747 & 1.9029 \\
\hline & & MSE & 1.2810 & 0.5783 & 0.2294 & 0.4569 & 0.1960 & 0.1593 & 0.2542 & 0.2031 & 0.1918 \\
\hline & & BISE & 0.8970 & 0.6719 & 0.3584 & 0.4770 & 0.3404 & 0.3246 & 0.3715 & 0.3474 & 0.3482 \\
\hline & $\left(0^{* 7}, 5,0^{* 7}\right)$ & $\mathrm{AE}$ & 2.2662 & 2.3913 & 2.0180 & 2.253 & 1.9643 & 1.8314 & 2.0685 & 1.9412 & 1.8650 \\
\hline & & MSE & 1.3089 & 0.6088 & 0.2177 & 0.4475 & 0.1890 & 0.1660 & 0.2400 & 0.1972 & 0.1940 \\
\hline & & BISE & 0.9167 & 0.7016 & 0.3540 & 0.4771 & 0.3374 & 0.3318 & 0.3669 & 0.3455 & 0.3526 \\
\hline \multirow[t]{3}{*}{$(20,20)$} & $\left(0^{* 20}\right)$ & $\mathrm{AE}$ & 2.2125 & 2.4063 & 2.0013 & 2.1899 & 1.9589 & 1.8529 & 2.0420 & 1.9407 & 1.8821 \\
\hline & & MSE & 1.1690 & 0.5822 & 0.1522 & 0.2929 & 0.1360 & 0.1236 & 0.1658 & 0.1401 & 0.1383 \\
\hline & & BISE & 0.8766 & 0.6717 & 0.3043 & 0.3934 & 0.2933 & 0.2876 & 0.3133 & 0.2990 & 0.3019 \\
\hline \multirow[t]{6}{*}{$(30,20)$} & $\left(10,0^{* 19}\right)$ & $\mathrm{AE}$ & 2.2809 & 2.378 & 1.9919 & 2.1764 & 1.9508 & 1.8479 & 2.0315 & 1.9332 & 1.8767 \\
\hline & & MSE & 1.4078 & 0.5683 & 0.1627 & 0.3018 & 0.1467 & 0.1338 & 0.1759 & 0.1509 & 0.1489 \\
\hline & & BISE & 0.9190 & 0.6626 & 0.3199 & 0.4080 & 0.3080 & 0.2993 & 0.3288 & 0.3128 & 0.3134 \\
\hline & $\left(2^{* 5}, 0^{* 15}\right)$ & $\mathrm{AE}$ & 2.1541 & 2.3328 & 1.9833 & 2.1673 & 1.9422 & 1.8397 & 2.0228 & 1.9245 & 1.8678 \\
\hline & & MSE & 1.1084 & 0.5502 & 0.1603 & 0.3005 & 0.1443 & 0.1331 & 0.1732 & 0.1490 & 0.1476 \\
\hline & & BISE & 0.8341 & 0.6503 & 0.3154 & 0.4003 & 0.3040 & 0.2999 & 0.3233 & 0.3093 & 0.3126 \\
\hline \multirow[t]{6}{*}{$(30,25)$} & $\left(5,0^{* 24}\right)$ & $\mathrm{AE}$ & 2.1418 & 2.3641 & 1.9555 & 2.1114 & 1.9211 & 1.8335 & 1.9896 & 1.9052 & 1.8569 \\
\hline & & MSE & 0.9984 & 0.5310 & 0.1276 & 0.2137 & 0.1199 & 0.1188 & 0.1342 & 0.1238 & 0.1267 \\
\hline & & BISE & 0.8118 & 0.6295 & 0.2909 & 0.3527 & 0.2837 & 0.2839 & 0.2960 & 0.2885 & 0.2926 \\
\hline & $\left(1^{* 5}, 0^{* 20}\right)$ & $\mathrm{AE}$ & 2.1309 & 2.3450 & 1.9522 & 2.1057 & 1.9181 & 1.8311 & 1.9860 & 1.9023 & 1.8542 \\
\hline & & MSE & 1.0569 & 0.5470 & 0.1285 & 0.2186 & 0.1196 & 0.1174 & 0.1353 & 0.1243 & 0.1272 \\
\hline & & BISE & 0.8122 & 0.6462 & 0.2811 & 0.3347 & 0.2767 & 0.2840 & 0.2837 & 0.2835 & 0.2924 \\
\hline \multirow[t]{3}{*}{$(30,30)$} & $\left(0^{* 30}\right)$ & $\mathrm{AE}$ & 2.1410 & 2.3809 & 1.9330 & 2.0675 & 1.9036 & 1.8282 & 1.9629 & 1.8894 & 1.8478 \\
\hline & & MSE & 0.9211 & 0.5411 & 0.0993 & 0.1524 & 0.0960 & 0.1007 & 0.1022 & 0.0995 & 0.1046 \\
\hline & & BISE & 0.7653 & 0.6337 & 0.2520 & 0.2974 & 0.2499 & 0.2603 & 0.2539 & 0.2554 & 0.2643 \\
\hline \multirow[t]{6}{*}{$(50,30)$} & $\left(20,0^{* 29}\right)$ & $\mathrm{AE}$ & 2.1354 & 2.3271 & 1.9470 & 2.0781 & 1.9179 & 1.8424 & 1.9763 & 1.9039 & 1.8626 \\
\hline & & MSE & 0.9733 & 0.5120 & 0.0988 & 0.1477 & 0.0951 & 0.0979 & 0.1020 & 0.0982 & 0.1022 \\
\hline & & BISE & 0.7933 & 0.6153 & 0.2524 & 0.2976 & 0.2487 & 0.2556 & 0.2552 & 0.2532 & 0.2604 \\
\hline & $\left(4^{* 5}, 0^{* 25}\right)$ & $\mathrm{AE}$ & 2.1266 & 2.3068 & 1.9366 & 2.0759 & 1.9060 & 1.8279 & 1.9675 & 1.8914 & 1.8484 \\
\hline & & MSE & 0.9804 & 0.5053 & 0.0995 & 0.1600 & 0.0954 & 0.0994 & 0.1034 & 0.0988 & 0.1035 \\
\hline & & BISE & 0.7752 & 0.6117 & 0.2583 & 0.3054 & 0.2546 & 0.2613 & 0.2608 & 0.2596 & 0.2664 \\
\hline \multirow[t]{6}{*}{$(50,40)$} & $\left(10,0^{* 39}\right)$ & $\mathrm{AE}$ & 2.0880 & 2.3274 & 1.9023 & 2.0050 & 1.8792 & 1.8180 & 1.9263 & 1.8670 & 1.8331 \\
\hline & & MSE & 0.7957 & 0.5074 & 0.0802 & 0.0944 & 0.0810 & 0.0905 & 0.0788 & 0.0848 & 0.0920 \\
\hline & & BISE & 0.7074 & 0.6132 & 0.2268 & 0.2377 & 0.2289 & 0.2462 & 0.2239 & 0.2348 & 0.2467 \\
\hline & $\left(2^{* 5}, 0^{* 35}\right)$ & $\mathrm{AE}$ & 2.0623 & 2.3327 & 1.8915 & 1.9953 & 1.8685 & 1.8077 & 1.9156 & 1.8564 & 1.8226 \\
\hline & & MSE & 0.7624 & 0.5135 & 0.0850 & 0.1039 & 0.0856 & 0.0953 & 0.0840 & 0.0893 & 0.0964 \\
\hline & & BISE & 0.6953 & 0.6180 & 0.2362 & 0.2537 & 0.2373 & 0.2533 & 0.2346 & 0.2429 & 0.2540 \\
\hline \multirow[t]{3}{*}{$(50,50)$} & $\left(0^{* 50}\right)$ & $\mathrm{AE}$ & 2.0701 & 2.3358 & 1.8730 & 1.9605 & 1.8535 & 1.8015 & 1.8937 & 1.8428 & 1.8138 \\
\hline & & MSE & 0.6934 & 0.5039 & 0.0690 & 0.0742 & 0.0713 & 0.0830 & 0.0665 & 0.0747 & 0.0824 \\
\hline & & BISE & 0.6726 & 0.6097 & 0.2164 & 0.2205 & 0.2196 & 0.2385 & 0.2124 & 0.2248 & 0.2368 \\
\hline
\end{tabular}


Table 5. Avrege estimates (AE), MSEs and baises of ML, LS and Bayes estimates under SE, LINEX and GE of $\lambda$

\begin{tabular}{|c|c|c|c|c|c|c|c|c|c|c|c|}
\hline \multirow[b]{2}{*}{$(n, m)$} & \multirow[b]{2}{*}{ Scheme } & & \multirow{2}{*}{ MLE } & \multirow[t]{2}{*}{ LSE } & \multirow[t]{2}{*}{$\overline{\mathrm{SEL}}$} & \multicolumn{3}{|c|}{ LINEX } & \multicolumn{3}{|c|}{$\overline{\mathrm{GE}}$} \\
\hline & & & & & & $c=-2$ & $c=0.5$ & $c=2$ & $q=-2$ & $q=0.5$ & $q=2$ \\
\hline \multirow[t]{9}{*}{$(20,15)$} & $\left(5,0^{* 14}\right)$ & $\overline{\mathrm{AE}}$ & 1.6776 & 0.8992 & 1.0100 & 1.0638 & 0.9965 & 0.9580 & 1.0361 & 0.9686 & 0.9274 \\
\hline & & MSE & 3.0885 & 0.1400 & 0.0784 & 0.0940 & 0.0756 & 0.0704 & 0.0804 & 0.0787 & 0.0829 \\
\hline & & Bias & 1.0787 & 0.3517 & 0.2237 & 0.2365 & 0.2216 & 0.2183 & 0.2231 & 0.2290 & 0.2379 \\
\hline & $\left(1^{* 5}, 0^{* 10}\right)$ & $\mathrm{AE}$ & 1.7680 & 0.9198 & 1.0064 & 1.0592 & 0.9932 & 0.9557 & 1.0319 & 0.966 & 0.9258 \\
\hline & & MSE & 3.2110 & 0.1285 & 0.0764 & 0.0918 & 0.0737 & 0.0687 & 0.0784 & 0.0767 & 0.0809 \\
\hline & & Bias & 1.1159 & 0.3351 & 0.2198 & 0.2323 & 0.2177 & 0.2146 & 0.2191 & 0.2248 & 0.2342 \\
\hline & $\left(0^{* 7}, 5,0^{* 7}\right)$ & $\mathrm{AE}$ & 1.6807 & 0.9070 & 1.0047 & 1.0591 & 0.9913 & 0.9533 & 1.031 & 0.9634 & 0.9224 \\
\hline & & MSE & 2.6909 & 0.1319 & 0.0795 & 0.0949 & 0.0768 & 0.0723 & 0.0811 & 0.0805 & 0.0854 \\
\hline & & Bias & 1.0548 & 0.3410 & 0.2263 & 0.2378 & 0.2242 & 0.2216 & 0.2253 & 0.2319 & 0.2424 \\
\hline \multirow[t]{3}{*}{$(20,20)$} & $\left(0^{* 20}\right)$ & $\mathrm{AE}$ & 1.6636 & 0.9065 & 1.0144 & 1.0652 & 1.0017 & 0.9653 & 1.0387 & 0.9762 & 0.9379 \\
\hline & & MSE & 2.6073 & 0.1295 & 0.0640 & 0.0790 & 0.0613 & 0.0562 & 0.0667 & 0.0627 & 0.0651 \\
\hline & & Bias & 1.0305 & 0.3409 & 0.2010 & 0.2155 & 0.1984 & 0.1940 & 0.2022 & 0.2028 & 0.2098 \\
\hline \multirow[t]{6}{*}{$(30,20)$} & $\left(10,0^{* 19}\right)$ & $\mathrm{AE}$ & 1.6264 & 0.9108 & 1.0259 & 1.0783 & 1.0128 & 0.9754 & 1.0504 & 0.9873 & 0.9487 \\
\hline & & MSE & 2.7119 & 0.1307 & 0.0698 & 0.0891 & 0.0661 & 0.0588 & 0.0738 & 0.0667 & 0.0675 \\
\hline & & Bias & 1.0094 & 0.3409 & 0.2082 & 0.2229 & 0.2055 & 0.2003 & 0.2089 & 0.2104 & 0.2162 \\
\hline & $\left(2^{* 5}, 0^{* 15}\right)$ & $\mathrm{AE}$ & 1.6866 & 0.9386 & 1.0358 & 1.0859 & 1.0231 & 0.9865 & 1.0594 & 0.9983 & 0.9604 \\
\hline & & MSE & 2.5830 & 0.1265 & 0.0747 & 0.0922 & 0.0711 & 0.0634 & 0.0783 & 0.0717 & 0.0721 \\
\hline & & Bias & 1.0181 & 0.3366 & 0.2127 & 0.2279 & 0.2097 & 0.2037 & 0.2142 & 0.2136 & 0.2193 \\
\hline \multirow[t]{6}{*}{$(30,25)$} & $\left(5,0^{* 24}\right)$ & $\mathrm{AE}$ & 1.6354 & 0.9181 & 1.0436 & 1.0930 & 1.0312 & 0.9956 & 1.0664 & 1.0076 & 0.9715 \\
\hline & & MSE & 2.4490 & 0.1281 & 0.0634 & 0.0816 & 0.0599 & 0.0524 & 0.0678 & 0.0592 & 0.0585 \\
\hline & & Bias & 0.9727 & 0.3382 & 0.1976 & 0.2152 & 0.1940 & 0.1857 & 0.2007 & 0.1955 & 0.1966 \\
\hline & $\left(1^{* 5}, 0^{* 20}\right)$ & $\mathrm{AE}$ & 1.6256 & 0.9335 & 1.0372 & 1.0857 & 1.0251 & 0.9905 & 1.0597 & 1.0020 & 0.9668 \\
\hline & & MSE & 2.282 & 0.1268 & 0.0583 & 0.0746 & 0.0552 & 0.0488 & 0.0622 & 0.0549 & 0.0546 \\
\hline & & Bias & 0.9475 & 0.3382 & 0.1880 & 0.2044 & 0.1848 & 0.1788 & 0.1908 & 0.1872 & 0.1908 \\
\hline \multirow[t]{3}{*}{$(30,30)$} & $\left(0^{* 30}\right)$ & $\mathrm{AE}$ & 1.5432 & 0.9121 & 1.0484 & 1.0952 & 1.0367 & 1.0028 & 1.0699 & 1.0146 & 0.9805 \\
\hline & & MSE & 1.9515 & 0.1270 & 0.0582 & 0.0753 & 0.0548 & 0.0475 & 0.0627 & 0.0537 & 0.0523 \\
\hline & & Bias & 0.8729 & 0.3371 & 0.1857 & 0.2043 & 0.1819 & 0.1746 & 0.1899 & 0.1832 & 0.1848 \\
\hline \multirow[t]{6}{*}{$(50,30)$} & $\left(20,0^{* 29}\right)$ & $\mathrm{AE}$ & 1.5764 & 0.9411 & 1.0426 & 1.0884 & 1.0312 & 0.9983 & 1.0636 & 1.0097 & 0.9767 \\
\hline & & MSE & 2.0837 & 0.1244 & 0.0574 & 0.0737 & 0.0541 & 0.0471 & 0.0613 & 0.0533 & 0.0521 \\
\hline & & Bias & 0.9074 & 0.3334 & 0.1815 & 0.1966 & 0.1786 & 0.1729 & 0.1842 & 0.1808 & 0.1840 \\
\hline & $\left(4^{* 5}, 0^{* 25}\right)$ & $\mathrm{AE}$ & 1.6012 & 0.9504 & 1.0506 & 1.0989 & 1.0386 & 1.0044 & 1.0727 & 1.0160 & 0.9817 \\
\hline & & MSE & 2.2220 & 0.1242 & 0.0546 & 0.0711 & 0.0515 & 0.0454 & 0.0588 & 0.0507 & 0.0501 \\
\hline & & Bias & 0.9225 & 0.3337 & 0.1841 & 0.2035 & 0.1803 & 0.1727 & 0.1886 & 0.1811 & 0.1829 \\
\hline \multirow[t]{6}{*}{$(50,40)$} & $\left(10,0^{* 39}\right)$ & $\mathrm{AE}$ & 1.4789 & 0.9349 & 1.0626 & 1.1079 & 1.0513 & 1.0191 & 1.0828 & 1.0312 & 0.9998 \\
\hline & & MSE & 1.4797 & 0.1203 & 0.0474 & 0.0648 & 0.0440 & 0.0367 & 0.0525 & 0.0417 & 0.0388 \\
\hline & & BISE & 0.7823 & 0.3304 & 0.1667 & 0.1894 & 0.1619 & 0.1507 & 0.1734 & 0.1589 & 0.1559 \\
\hline & $\left(2^{* 5}, 0^{* 35}\right)$ & $\mathrm{AE}$ & 1.5159 & 0.9334 & 1.0702 & 1.1148 & 1.0591 & 1.0268 & 1.0901 & 1.0392 & 1.0080 \\
\hline & & MSE & 1.5961 & 0.1207 & 0.0532 & 0.0711 & 0.0494 & 0.0407 & 0.0584 & 0.0470 & 0.0433 \\
\hline & & BISE & 0.8005 & 0.3305 & 0.1735 & 0.1937 & 0.1693 & 0.1596 & 0.1789 & 0.1682 & 0.1661 \\
\hline \multirow[t]{3}{*}{$(50,50)$} & $\left(0^{* 50}\right)$ & $\mathrm{AE}$ & 1.4424 & 0.9294 & 1.0719 & 1.1131 & 1.0617 & 1.0324 & 1.0903 & 1.0436 & 1.0153 \\
\hline & & MSE & 1.4126 & 0.1225 & 0.0441 & 0.0588 & 0.0411 & 0.0342 & 0.0485 & 0.0388 & 0.0357 \\
\hline & & Bias & 0.7359 & 0.333 & 0.1593 & 0.1802 & 0.1551 & 0.1456 & 0.1656 & 0.1529 & 0.1506 \\
\hline
\end{tabular}


Table 6. Avrege estimates (AE), MSEs and baises of ML, LS and Bayes estimates under SE, LINEX and GE of $\beta$

\begin{tabular}{|c|c|c|c|c|c|c|c|c|c|c|c|}
\hline \multirow[b]{2}{*}{$(n, m)$} & \multirow[b]{2}{*}{ Scheme } & & \multirow[t]{2}{*}{ MLE } & \multirow[t]{2}{*}{ LSE } & \multirow[t]{2}{*}{ SEL } & \multicolumn{3}{|c|}{ LINEX } & \multicolumn{3}{|c|}{ GE } \\
\hline & & & & & & $c=-2$ & $c=0.5$ & $c=2$ & $q=-2$ & $q=0.5$ & $q=2$ \\
\hline \multirow[t]{9}{*}{$(20,15)$} & $\left(5,0^{* 14}\right)$ & $\mathrm{AE}$ & 2.6570 & 1.9943 & 1.9332 & 2.1718 & 1.8719 & 1.7156 & 1.9928 & 1.8384 & 1.7415 \\
\hline & & MSE & 2.0229 & 0.4102 & 0.3313 & 0.5118 & 0.3020 & 0.2932 & 0.3429 & 0.3284 & 0.3479 \\
\hline & & Bias & 0.9588 & 0.5455 & 0.4474 & 0.5023 & 0.4409 & 0.4585 & 0.4436 & 0.4622 & 0.4906 \\
\hline & $\left(1^{* 5}, 0^{* 10}\right)$ & $\mathrm{AE}$ & 2.7998 & 2.0562 & 1.9608 & 2.2004 & 1.8993 & 1.7416 & 2.0199 & 1.8668 & 1.7711 \\
\hline & & MSE & 2.5753 & 0.3998 & 0.3478 & 0.5387 & 0.3168 & 0.2974 & 0.3622 & 0.3408 & 0.3555 \\
\hline & & Bias & 1.0584 & 0.5334 & 0.4627 & 0.5313 & 0.4539 & 0.4641 & 0.4616 & 0.4743 & 0.4999 \\
\hline & $\left(0^{* 7}, 5,0^{* 7}\right)$ & $\mathrm{AE}$ & 2.7794 & 2.0421 & 1.9311 & 2.1764 & 1.8684 & 1.7089 & 1.9920 & 1.8343 & 1.7356 \\
\hline & & MSE & 2.5326 & 0.3998 & 0.3482 & 0.5374 & 0.3177 & 0.3063 & 0.3608 & 0.3446 & 0.3641 \\
\hline & & Bias & 1.0397 & 0.5383 & 0.4675 & 0.5241 & 0.4618 & 0.4768 & 0.4624 & 0.4849 & 0.5135 \\
\hline \multirow[t]{3}{*}{$(20,20)$} & $\left(0^{* 20}\right)$ & $\mathrm{AE}$ & 2.6756 & 2.0689 & 1.9324 & 2.1672 & 1.8733 & 1.7252 & 1.9895 & 1.8426 & 1.7522 \\
\hline & & MSE & 2.1414 & 0.3589 & 0.3083 & 0.5057 & 0.2790 & 0.2708 & 0.3231 & 0.3009 & 0.3156 \\
\hline & & Bias & 0.9467 & 0.5041 & 0.4318 & 0.4996 & 0.4243 & 0.4384 & 0.4306 & 0.4438 & 0.4679 \\
\hline \multirow[t]{6}{*}{$(30,20)$} & $\left(10,0^{* 19}\right)$ & $\mathrm{AE}$ & 2.5772 & 2.0661 & 1.9771 & 2.186 & 1.9250 & 1.7899 & 2.0270 & 1.8991 & 1.8201 \\
\hline & & MSE & 1.6961 & 0.3575 & 0.2787 & 0.4375 & 0.2538 & 0.2367 & 0.2922 & 0.2688 & 0.2748 \\
\hline & & Bias & 0.8405 & 0.5013 & 0.4079 & 0.4752 & 0.4012 & 0.4111 & 0.4082 & 0.4165 & 0.4356 \\
\hline & $\left(2^{* 5}, 0^{* 15}\right)$ & $\mathrm{AE}$ & 2.5737 & 2.0443 & 1.9732 & 2.1770 & 1.9223 & 1.7885 & 2.0223 & 1.8967 & 1.8194 \\
\hline & & MSE & 1.5318 & 0.3430 & 0.2641 & 0.4019 & 0.2429 & 0.2254 & 0.2754 & 0.2569 & 0.2631 \\
\hline & & Bias & 0.8126 & 0.4953 & 0.4017 & 0.4604 & 0.3944 & 0.3986 & 0.4020 & 0.4086 & 0.4237 \\
\hline \multirow[t]{6}{*}{$(30,25)$} & $\left(5,0^{* 24}\right)$ & $\mathrm{AE}$ & 2.5120 & 2.0299 & 1.9462 & 2.1399 & 1.8987 & 1.7745 & 1.9930 & 1.8737 & 1.8007 \\
\hline & & MSE & 1.2705 & 0.3020 & 0.2348 & 0.3509 & 0.2200 & 0.2170 & 0.2418 & 0.2340 & 0.2461 \\
\hline & & Bias & 0.7472 & 0.4605 & 0.3793 & 0.4299 & 0.3755 & 0.3883 & 0.3776 & 0.3892 & 0.4082 \\
\hline & $\left(1^{* 5}, 0^{* 20}\right)$ & $\mathrm{AE}$ & 2.4906 & 2.0287 & 1.9235 & 2.1063 & 1.8791 & 1.7610 & 1.9679 & 1.8548 & 1.7853 \\
\hline & & MSE & 1.2145 & 0.3061 & 0.2231 & 0.3160 & 0.2128 & 0.2149 & 0.2271 & 0.2258 & 0.2397 \\
\hline & & Bias & 0.7349 & 0.4561 & 0.3784 & 0.4170 & 0.3769 & 0.3937 & 0.3749 & 0.3908 & 0.4118 \\
\hline \multirow[t]{3}{*}{$(30,30)$} & $\left(0^{* 30}\right)$ & $\mathrm{AE}$ & 2.4705 & 2.0437 & 1.9456 & 2.1225 & 1.9028 & 1.7886 & 1.9880 & 1.8802 & 1.8143 \\
\hline & & MSE & 1.1384 & 0.2646 & 0.2122 & 0.3012 & 0.2015 & 0.1983 & 0.2170 & 0.2126 & 0.2228 \\
\hline & & Bias & 0.6939 & 0.4197 & 0.3600 & 0.4044 & 0.3568 & 0.3683 & 0.3591 & 0.3686 & 0.3848 \\
\hline \multirow[t]{6}{*}{$(50,30)$} & $\left(20,0^{* 29}\right)$ & $\mathrm{AE}$ & 2.4136 & 2.0473 & 1.9555 & 2.1168 & 1.9177 & 1.8177 & 1.9929 & 1.8987 & 1.8423 \\
\hline & & MSE & 0.8308 & 0.2772 & 0.1742 & 0.2613 & 0.1644 & 0.1612 & 0.1798 & 0.1721 & 0.1779 \\
\hline & & Bias & 0.6243 & 0.4338 & 0.3329 & 0.3738 & 0.3288 & 0.3356 & 0.3326 & 0.3378 & 0.3496 \\
\hline & $\left(4^{* 5}, 0^{* 25}\right)$ & $\mathrm{AE}$ & 2.4151 & 2.0426 & 1.9660 & 2.1136 & 1.9301 & 1.8329 & 2.0011 & 1.9121 & 1.8579 \\
\hline & & MSE & 0.8256 & 0.2493 & 0.1688 & 0.2288 & 0.1496 & 0.1448 & 0.1645 & 0.1555 & 0.1592 \\
\hline & & Bias & 0.6243 & 0.4097 & 0.3177 & 0.3532 & 0.3137 & 0.3191 & 0.3178 & 0.3217 & 0.3315 \\
\hline \multirow[t]{6}{*}{$(50,40)$} & $\left(10,0^{* 39}\right)$ & $\mathrm{AE}$ & 2.3628 & 2.0409 & 1.9593 & 2.0971 & 1.9262 & 1.8362 & 1.9919 & 1.9096 & 1.8598 \\
\hline & & MSE & 0.7313 & 0.2460 & 0.1603 & 0.2273 & 0.1573 & 0.1520 & 0.1696 & 0.1632 & 0.1667 \\
\hline & & BISE & 0.5797 & 0.4045 & 0.3220 & 0.3561 & 0.3187 & 0.3256 & 0.3222 & 0.3264 & 0.3368 \\
\hline & $\left(2^{* 5}, 0^{* 35}\right)$ & $\mathrm{AE}$ & 2.3662 & 2.0362 & 1.9447 & 2.0776 & 1.9128 & 1.8266 & 1.9764 & 1.8963 & 1.8481 \\
\hline & & MSE & 0.6728 & 0.2178 & 0.1468 & 0.1984 & 0.1409 & 0.1418 & 0.1498 & 0.1470 & 0.1530 \\
\hline & & BISE & 0.5650 & 0.3750 & 0.3008 & 0.3275 & 0.3003 & 0.3120 & 0.2986 & 0.3084 & 0.3208 \\
\hline \multirow[t]{3}{*}{$(50,50)$} & $\left(0^{* 50}\right)$ & $\mathrm{AE}$ & 2.3126 & 2.0188 & 1.9303 & 2.0546 & 1.9007 & 1.8196 & 1.9600 & 1.8852 & 1.8401 \\
\hline & & MSE & 0.5801 & 0.1960 & 0.1383 & 0.1801 & 0.1340 & 0.1354 & 0.1401 & 0.1394 & 0.1451 \\
\hline & & Bias & 0.5254 & 0.3573 & 0.2988 & 0.3249 & 0.2969 & 0.3052 & 0.2979 & 0.3036 & 0.3134 \\
\hline
\end{tabular}


Table 7. Average lengths and coverage probabilities of $95 \%$ confidence intervals of $\alpha, \lambda$ and $\beta$.

\begin{tabular}{|c|c|c|c|c|c|c|}
\hline$(n, m)$ & Scheme & & MLE & Bayes & Boot b & Boot t \\
\hline \multirow[t]{9}{*}{$(20,15)$} & $\left(5,0^{* 14}\right)$ & $\alpha$ & $4.5470(0.915)$ & $1.6406(0.919)$ & $3.5957(0.962)$ & $2.4975(0.919)$ \\
\hline & & $\lambda$ & $3.3056(0.967)$ & $0.7820(0.942)$ & $4.6724(0.974)$ & $2.5782(0.964)$ \\
\hline & & $\beta$ & $4.7568(0.964)$ & $1.7246(0.911)$ & $5.4260(0.963)$ & $2.6727(0.957)$ \\
\hline & $\left(1^{* 5}, 0^{* 10}\right)$ & $\alpha$ & $4.2780(0.899)$ & $1.6276(0.915)$ & $3.4129(0.981)$ & $2.4918(0.925)$ \\
\hline & & $\lambda$ & $3.4608(0.974)$ & $0.7704(0.940)$ & $4.6729(0.970)$ & $2.5665(0.969)$ \\
\hline & & $\beta$ & $5.0032(0.962)$ & $1.7337(0.907)$ & $4.9885(0.978)$ & $2.6625(0.964)$ \\
\hline & $\left(0^{* 7}, 5,0^{* 7}\right)$ & $\alpha$ & $4.3934(0.899)$ & $1.6636(0.924)$ & $3.3041(0.975)$ & 2.4911 (0.917) \\
\hline & & $\lambda$ & $3.2932(0.961)$ & 0.7854 (0.939) & $4.6568(0.992)$ & $2.5602(0.962)$ \\
\hline & & $\beta$ & $4.8538(0.963)$ & $1.7508(0.917)$ & $4.8616(0.967)$ & $2.6253(0.970)$ \\
\hline \multirow[t]{3}{*}{$(20,20)$} & $\left(0^{* 20}\right)$ & $\alpha$ & $4.3073(0.906)$ & $1.4827(0.942)$ & $3.3180(0.952)$ & $2.4904(0.929)$ \\
\hline & & $\lambda$ & $3.2752(0.973)$ & $0.7575(0.961)$ & $4.4673(0.961)$ & $2.5434(0.959)$ \\
\hline & & $\beta$ & $4.6645(0.972)$ & $1.6972(0.923)$ & $4.6179(0.962)$ & $2.6275(0.961)$ \\
\hline \multirow[t]{6}{*}{$(30,20)$} & $\left(10,0^{* 19}\right)$ & $\alpha$ & $4.3788(0.890)$ & $1.4591(0.911)$ & $3.3258(0.950)$ & $2.4911(0.964)$ \\
\hline & & $\lambda$ & $3.1833(0.952)$ & $0.7679(0.947)$ & $4.4232(0.962)$ & $2.5417(0.981)$ \\
\hline & & $\beta$ & $4.2777(0.964)$ & $1.6174(0.938)$ & $4.4906(0.966)$ & $2.6545(0.968)$ \\
\hline & $\left(2^{* 5}, 0^{* 15}\right)$ & $\alpha$ & $4.1536(0.901)$ & $1.4619(0.917)$ & $3.2679(0.949)$ & $2.5027(0.936)$ \\
\hline & & $\lambda$ & $3.3157(0.970)$ & $0.7585(0.930)$ & $4.4692(0.987)$ & $2.5418(0.974)$ \\
\hline & & $\beta$ & $4.2681(0.962)$ & $1.6008(0.901)$ & $4.0636(0.964)$ & $2.5863(0.961)$ \\
\hline \multirow[t]{6}{*}{$(30,25)$} & $\left(5,0^{* 24}\right)$ & $\alpha$ & $4.1187(0.915)$ & $1.3493(0.927)$ & $3.1057(0.958)$ & 2.4899 (0.927) \\
\hline & & $\lambda$ & $3.2203(0.971)$ & $0.7491(0.936)$ & $3.9955(0.973)$ & $2.5419(0.968)$ \\
\hline & & $\beta$ & $4.1224(0.961)$ & $1.5581(0.907)$ & $3.8352(0.965)$ & $2.6071(0.960)$ \\
\hline & $\left(1^{* 5}, 0^{* 20}\right)$ & $\alpha$ & $4.0925(0.898)$ & $1.3412(0.928)$ & $3.0016(0.978)$ & 2.4754 (0.924) \\
\hline & & $\lambda$ & $3.2048(0.965)$ & $0.7452(0.945)$ & $3.8093(0.982)$ & $2.5417(0.962)$ \\
\hline & & $\beta$ & $4.0082(0.963)$ & $1.5222(0.915)$ & $3.6697(0.933)$ & $2.5608(0.963)$ \\
\hline \multirow[t]{3}{*}{$(30,30)$} & $\left(0^{* 30}\right)$ & $\alpha$ & $4.0848(0.903)$ & $1.2563(0.921)$ & $2.9635(0.951)$ & $2.4604(0.925)$ \\
\hline & & $\lambda$ & $3.0374(0.963)$ & $0.7308(0.939)$ & $3.8924(0.968)$ & $2.5413(0.975)$ \\
\hline & & $\beta$ & $3.9388(0.971)$ & $1.5052(0.948)$ & $3.4685(0.966)$ & $2.5599(0.967)$ \\
\hline \multirow[t]{6}{*}{$(50,30)$} & $\left(20,0^{* 29}\right)$ & $\alpha$ & $4.1107(0.912)$ & $1.2497(0.928)$ & $3.0522(0.965)$ & $2.4675(0.935)$ \\
\hline & & $\lambda$ & $3.1088(0.972)$ & $0.7240(0.939)$ & $3.9205(0.976)$ & $2.5412(0.968)$ \\
\hline & & $\beta$ & $3.6136(0.973)$ & $1.4301(0.918)$ & $3.5869(0.962)$ & $2.6506(0.971)$ \\
\hline & $\left(4^{* 5}, 0^{* 25}\right)$ & $\alpha$ & $4.0669(0.891)$ & $1.2713(0.930)$ & $2.9996(0.950)$ & $2.4762(0.926)$ \\
\hline & & $\lambda$ & $3.1676(0.972)$ & $0.7367(0.938)$ & $3.8249(0.953)$ & $2.5409(0.981)$ \\
\hline & & $\beta$ & $3.5519(0.971)$ & $1.381(0.913)$ & $3.1727(0.964)$ & $2.5368(0.957)$ \\
\hline \multirow[t]{6}{*}{$(50,40)$} & $\left(10,0^{* 39}\right)$ & $\alpha$ & $3.9888(0.915)$ & $1.1251(0.926)$ & $2.8057(0.950)$ & $2.4527(0.933)$ \\
\hline & & $\lambda$ & $2.9140(0.967)$ & $0.7191(0.953)$ & $3.5513(0.955)$ & $2.5322(0.967)$ \\
\hline & & $\beta$ & $3.2893(0.963)$ & $1.3359(0.905)$ & $2.9323(0.961)$ & $2.5529(0.968)$ \\
\hline & $\left(2^{* 5}, 0^{* 35}\right)$ & $\alpha$ & $3.9329(0.907)$ & $1.1182(0.918)$ & $2.7248(0.948)$ & $2.4620(0.944)$ \\
\hline & & $\lambda$ & $2.9859(0.966)$ & $0.7126(0.947)$ & $3.4529(0.956)$ & $2.5107(0.984)$ \\
\hline & & $\beta$ & $3.2600(0.968)$ & $1.3108(0.899)$ & $2.8150(0.961)$ & $2.5134(0.959)$ \\
\hline \multirow[t]{3}{*}{$(50,50)$} & $\left(0^{* 50}\right)$ & $\alpha$ & $3.8920(0.911)$ & $1.0304(0.922)$ & $2.6496(0.961)$ & $2.4417(0.935)$ \\
\hline & & $\lambda$ & $2.8598(0.974)$ & $0.6915(0.943)$ & $3.2173(0.959)$ & $2.4924(0.948)$ \\
\hline & & $\beta$ & $3.1196(0.971)$ & $1.2760(0.907)$ & $2.5091(0.952)$ & $2.4678(0.965)$ \\
\hline
\end{tabular}

